Article

\title{
Duodenal Metatranscriptomics to Define Human and Microbial Functional Alterations Associated with Severe Obesity: A Pilot Study
}

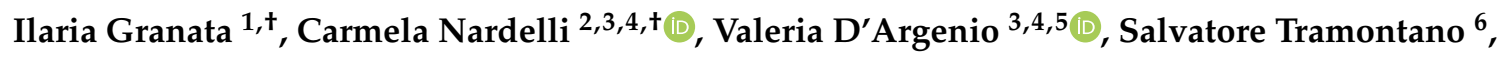 \\ Debora Compare $^{7}$, Mario Rosario Guarracino ${ }^{8}\left({ }^{1}\right.$, Gerardo Nardone ${ }^{7}$, Vincenzo Pilone ${ }^{6}$ \\ and Lucia Sacchetti ${ }^{3,4, *}$ \\ 1 Institute for High Performance Computing and Networking (ICAR), National Research Council (CNR), \\ 80131 Naples, Italy; ilaria.granata@icar.cnr.it \\ 2 Department of Molecular Medicine and Medical Biotechnologies, University of Naples Federico II, \\ 80131 Naples, Italy; carmela.nardelli@unina.it \\ 3 CEINGE Biotecnologie Avanzate S.C.a R.L., 80131 Naples, Italy; dargenio@ceinge.unina.it \\ 4 Task Force on Microbiome Studies, University of Naples Federico II, 80131 Naples, Italy \\ 5 Department of Human Sciences and Quality of Life Promotion, San Raffaele Open University, \\ 00166 Rome, Italy \\ 6 Department of Medicine and Surgery, University of Salerno, 84084 Salerno, Italy; salvytra@libero.it (S.T.); \\ vpilone@unisa.it (V.P.) \\ 7 Department of Clinical Medicine and Surgery, University of Naples Federico II, 80131 Naples, Italy; \\ debora.compare@unina.it (D.C.); gerardoantoniopio.nardone@unina.it (G.N.) \\ 8 Department of Economics and Law, University of Cassino and Southern Lazio, 03043 Cassino, Italy; \\ mario.guarracino@unicas.it \\ * Correspondence: sacchett@unina.it \\ $\dagger$ These authors contributed equally to this work.
}

Received: 22 October 2020; Accepted: 16 November 2020; Published: 17 November 2020

\begin{abstract}
Obesity is a multifactorial disorder, and the gut microbiome has been suggested to contribute to its onset. In order to better clarify the role of the microbiome in obesity, we evaluated the metatranscriptome in duodenal biopsies from a cohort of 23 adult severely obese and lean control subjects using next generation sequencing. Our aim was to provide a general picture of the duodenal metatranscriptome associated with severe obesity. We found altered expressions of human and microbial genes in the obese compared to lean subjects, with most of the gene alterations being present in the carbohydrate, protein, and lipid metabolic pathways. Defects were also present in several human genes involved in epithelial intestinal cells differentiation and function, as well as in the immunity/inflammation pathways. Moreover, the microbial taxa abundance inferred by our transcriptomic data differed in part from the data that we previously evaluated by $16 \mathrm{~S}$ rRNA in 13/23 individuals of our cohort, particularly concerning the Firmicutes and Proteobacteria phyla abundances. In conclusion, our pilot study provides the first taxonomic and functional characterization of duodenal microbiota in severely obese subjects and lean controls. Our findings suggest that duodenal microbiome and human genes both play a role in deregulating metabolic pathways, likely affecting energy metabolism and thus contributing to the obese phenotype.
\end{abstract}

Keywords: metatranscriptomics; microbial-host duodenal transcriptome; human obesity; metabolism

\section{Introduction}

Obesity is a pandemic disease whose prevalence has dramatically increased since the 1970s. Linear time trend forecasts indicate that, by $2030,51 \%$ of the world population will be obese [1]. 
Notably, the number of severely obese individuals (body mass index (BMI) $\geq 40 \mathrm{~kg} / \mathrm{m}^{2}$ ) has increased faster than that of obese subjects $\left(B M I \geq 30 \mathrm{~kg} / \mathrm{m}^{2}\right)$ [1]. Obesity is a risk factor for metabolic disorders, autoimmune diseases, and cancer [2-4], and dramatically impacts healthcare costs [5]. Furthermore, severely obese subjects have a much greater risk for diabetes and other diseases than mildly obese subjects and are predicted to increase healthcare costs in the future [6]. A better understanding of the pathogenic factors involved in the onset and progression of obesity may lead to interventions to reduce its spread and contain the related costs. Except for monogenic forms of obesity accounting for only $5 \%$ of severe obesity cases [7], obesity is driven by genetics, epigenetics, lifestyle, and the environment, each of which impacts the energy balance [8-11].

In the last two decades, the gut microbiota has emerged as a contributor to obesity and obesity-associated diseases [2,4,12-14]. The human microbiota inhabits the surfaces and niches of our organism and consists of a variety of microbes, mainly bacteria, but also fungi, viruses, and archaea, equal in number to human cells (1.3:1) [15]. In detail, the gut microbiota, and its approximately 3.3 million genes (the "microbiome") [13], interact with the human genome and play a role in several functions, particularly in digesting food, preserving the gut mucosal barrier integrity, and priming the immune system, thus globally contributing to human health $[16,17]$. Thanks to the advent of next generation sequencing (NGS) techniques in the early 2000s [18], together with that of powerful tools for the bioinformatics processing of sequencing data $[19,20]$, it has become possible to explore the gut microbiota composition in association with obesity [12,21-24]. However, given the large intra- and interindividual variability of the gut microbiota, about $20 \%$ of which is attributable to environmental factors and BMI [25], it is difficult to establish whether a microbiota is a "healthy" or "obese" one. Furthermore, metagenomically defined abundant microorganisms in the gut were found to be inactive or dormant [26]. Therefore, it is now evident that, to determine the role of the microbiome in human health or disease, its activity, as well as its composition, must be evaluated [27]. The gut microbiome has been mostly investigated in feces due to the ease of sampling [2,12-14], but the microbial community differs between the upper and lower intestinal tract [28]. Indeed, comparisons between the global bacterial assemblages at the phylotype level across eight different regions of the gastrointestinal tract revealed that the global bacterial structures differ significantly among regions [29]. In addition, the human small intestine is largely involved in nutrient digestion and absorption. In fact, between $85 \%$ and $95 \%$ of protein intake is digested in this tract, which is also important for simple carbohydrate metabolism and central metabolism [30-32]. We conducted a duodenal metatranscriptomics study in a cohort of adult severely obese (OB) and lean control subjects (CO) to identify obesity-associated alterations of both microbial and human gene expression, as well as their putative dysfunctional cross-regulation of metabolism.

\section{Materials and Methods}

\subsection{Patients and Controls}

In this study, we enrolled 12 severely obese patients (OB; 6 women and 6 men) and 11 lean control subjects (CO; 5 women and 6 men). Patients and controls were enrolled at the Surgery and Medicine Departments of the University of Salerno and of the University of Naples Federico II. Obese patients were selected among candidates for bariatric surgery and normal-weight controls were selected from among patients with gastroesophageal reflux symptoms. Inclusion criteria were an age range of 18-65 years, the filling of a weekly dietary scheme to examine specific behavioral items, and $\mathrm{BMI} \geq 40 \mathrm{Kg} / \mathrm{m}^{2}$ or $20.0-24.9 \mathrm{Kg} / \mathrm{m}^{2}$ for obese and control subjects, respectively. Exclusion criteria were diabetes, tumors, inflammatory bowel diseases, Crohn's disease, viral hepatitis, pharmacological treatment (i.e., antibiotics, pro- and prebiotics, antiviral or corticosteroid medications, or proton-pump inhibitors) in the last 2 months before sample collection. All patients underwent gastrointestinal endoscopy for diagnostic purposes and were enrolled after a histological examination revealed that they had a normal mucosa. The study was approved by the Ethics Committees of the Universities of 
Salerno and Naples (authorization n.50, 15/07/2015; amendment n. 141070, 26/11/2019 and n. 193/06, October 25, 2006; amendment n. 193/06/ESES1, October 1, 2014, respectively). All enrolled subjects gave their informed consent to participate in the study, which was carried out according to the Helsinki Declaration (2013). The clinical and anamnestic data of each subject were collected by a clinician, whereas dietary habits were self-reported and recorded by a nutritionist. Thirteen subjects $(7 \mathrm{OB}$ and $6 \mathrm{CO}$ ), belonging to our cohort, had previously been investigated by our team using $16 \mathrm{~S}$ rRNA sequencing to characterize their duodenal microbiome composition [24].

\subsection{Sample Collection}

We collected blood samples and duodenal biopsy specimens from all enrolled individuals. The biopsy sample was taken during an upper gastrointestinal endoscopy performed for diagnostic testing, under sterile conditions to avoid contamination, as reported elsewhere [24]. Biopsies were immediately stored in a vial containing RNA Later, then cooled in dry ice and stored at $-80{ }^{\circ} \mathrm{C}$ until RNA isolation for metatranscriptomics analysis. The patients' main biochemical parameters (Table S1) were evaluated by routine assays using the ACHITECT i2000R System (Abbott Laboratories, Wiesbaden, Germany).

\subsection{RNA Isolation and Sequencing}

Total RNA was obtained from each duodenal sample. Single biopsies $(10 \mathrm{mg} / \mathrm{each})$ were pulverized to a fine powder with a standard liquid nitrogen pre-chilled mortar and pestle. This powder was transferred to a $1.5 \mathrm{~mL}$ tube with $500 \mu \mathrm{L}$ of TRI Reagent solution (Ambion, Austin, TX, USA). The homogenate was vortexed vigorously for $10 \mathrm{~min}$ on a REAX 2000 (Heidolph, Apeldoorn, The Netherlands). After a quick spin-down, $200 \mu \mathrm{L}$ of chloroform was added to the homogenate, vortexed for $15 \mathrm{~s}$, and kept at room temperature for $5 \mathrm{~min}$. The partly separated mixture was centrifuged for $15 \mathrm{~min}$ at $10,000 \times \mathrm{g}$. The aqueous phase was transferred to a new $1.5 \mathrm{~mL}$ tube. RNA was purified by column precipitation, according to the mirVanaTM miRNA isolation kit (Ambion, Austin, TX, USA). At the end of the procedure, total RNA was eluted in $70 \mu \mathrm{L}$ of $95^{\circ} \mathrm{C}$ pre-warmed, nuclease-free water. Together with the duodenal samples, we processed a water sample as blank extraction control. The RNAs were quantified with the NanoDrop ND-1000 UV-Vis spectrophotometer (NanoDrop Technologies, Wilmington, DE, USA). The RNA extracts were further treated with DNase I (Qiagen, Hilden, Germany) to ensure the absence of residual DNA.

The quality and integrity of RNAs and the absence of residual DNA were assessed on the TapeStation (Agilent Technologies, Santa Clara, CA, USA) before library preparation. All samples had an RNA integrity number ranging between $6.6-8.7$, which indicates good-quality samples. Starting from an RNA input/sample of $800 \mathrm{ng}$, we first depleted human and bacterial ribosomal RNAs (rRNA), followed by a cleanup using Agencourt RNAClean XP beads (Beckman Coulter, Inc., Indianapolis, IN, USA) according to standard procedures (Ribo-Zero Gold Epidemiology protocol, Illumina, San Diego, CA, USA). The quality and quantity of depleted RNA samples were checked on the TapeStation. Then, we prepared an RNA library for each depleted RNA sample using the TruSeq RNA Sample Preparation kit v2 (Illumina, Inc., San Diego, CA, USA) according to the manufacturer's protocol. In detail, RNA samples were fragmented at $94{ }^{\circ} \mathrm{C}$ for $8 \mathrm{~min}$ on a thermal cycler. First-strand cDNA synthesis was performed at $25{ }^{\circ} \mathrm{C}$ for $10 \mathrm{~min}$, at $42{ }^{\circ} \mathrm{C}$ for $15 \mathrm{~min}$, and at $70{ }^{\circ} \mathrm{C}$ for $15 \mathrm{~min}$ using random hexamers and the SuperScript II Reverse Transcriptase (Thermo Fisher Scientific Inc., Waltham, MA, USA). Next, during second-strand cDNA synthesis, the RNA templates were removed, and a second replacement strand was generated by incorporation of dUTP (in place of dTTP, to keep strand information) to generate ds cDNA. AMPure XP beads (Beckman Coulter, Inc., Indianapolis, IN, USA) were used to clean up the blunt-ended cDNAs from the second-strand reaction mix. The $3^{\prime}$ ends of the ds cDNAs were then adenylated to facilitate adaptor ligation during the next step. After ligation of the indexing adaptors, AMPure XP beads were used to clean up the libraries a second time. Next, a PCR amplification step $\left(15\right.$ cycles of $98^{\circ} \mathrm{C}$ for $10 \mathrm{~s}, 60^{\circ} \mathrm{C}$ for $30 \mathrm{~s}$, and $72{ }^{\circ} \mathrm{C}$ for $30 \mathrm{~s}$ ) was used to selectively 
enrich the DNA fragments that had adapter molecules on both their ends and to amplify the amount of DNA in the library. Twelve libraries (from 6 obese patients and 6 controls) were pooled to obtain 2 pools (12 samples each) for cluster generation on the cBot using the TruSeq PE Cluster Kit v3 (Illumina, Inc., San Diego, CA, USA). Finally, sequencing was performed by loading each pool in 4 lanes of a single flow cell (24 samples per run) using the TruSeq SBS kit v3, 200 cycle PE, following the manufacturer's protocols (Illumina, HiSeq1500). The concentration of library pools for sequencing was $6 \mathrm{pM}$. All the technical controls used from RNA isolation to its sequencing, according to the manufacturers' instructions, are detailed in the Supplementary File 1.

\subsection{Metatranscriptomics Analysis/Processing}

Metatranscriptomics sequencing data were quality controlled by using FastQC v0.11.2 [33] and subsequently deprived of adapters and low-quality reads/ends by Trimmomatic v0.38 [34].

\subsubsection{Human Transcriptome Analysis}

Good-quality reads were aligned to the human reference genome (NCBI GRCh38) with Bowtie2 aligner v2.3.4.2 [35]. The abundances of host transcripts were quantified using the Kallisto tool v0.45.0 [36] and the counts were given as input to DESeq2 v1.24.0 [37] to determine the differential expression between the two groups under study. The genes were considered significantly different if the Logarithm of fold change ratio (Log2FC) $\geq|1|$ and Benjamini-Hochberg $(\mathrm{BH})$ adjusted $p$-value $\leq 0.05$.

\subsubsection{Bacterial Transcriptome Analysis}

The reads that failed to map to the host genome were aligned to the phiX vector sequences using the Bowtie2 tool and, once merged, to rRNA and tRNA sequences using the SortMeRNA v2.1 tool [38]. Non-rRNA and non-tRNA sequences, theoretically corresponding to putative mRNA, were used to annotate the microbial genes and pathways. In detail, the DIAMOND program v0.9.22 [39], a superfast BLAST-based algorithm, was used to map the reads to the NCBI nonredundant bacterial protein (RefSeq_bac) and to the SEED subsystem databases (ftp://ftp.theseed.org/subsystems/) with the "BLASTX" option. One hit for each read was retained, and hits with an e-value exceeding 1e-04 or a percentage of identical matches $\leq 97 \%$ were discarded. The RefSeq hits were then aggregated by organism or function annotations using the python script "DIAMOND_analysis_counter.py" tool, while the SEED subsystem hierarchy for SEED hits was reconstructed using the python script "DIAMOND_subsystems_analysis_counter.py," both obtained from SAMSA2 v2.2.0 pipeline [40]. In order to focus the analysis on functions linked to specific organisms of interest, we restricted the function annotations to the differentially abundant organisms, since each read processed by SAMSA software is provided by both an organism and functional annotation from the RefSeq database. This step was achieved through the python script "DIAMOND_specific_organism_retreiver.py." We evaluated the differential gene expression using DESeq2 v1.24.0 on the abundance tables for organism and function RefSeq annotations, as well as for each of the 4 SEED subsystem level hierarchies. Terms were considered significantly different if $\log 2 \mathrm{FC} \geq|1|$ and $\mathrm{BH}$-adjusted $p$-value $\leq 0.05$. Furthermore, the mRNA reads were mapped to the UniRef90 database using the HUMAnN $2.0 \mathrm{v} 2.7 .8$ tool [41] and the abundances obtained were combined into structured pathways from MetaCyc [42]. To identify the metabolic pathways specifically associated with obesity, we assessed the abundance of each feature in obese patients versus controls using generalized linear models in MaAsLin2 (Multivariate Association with Linear Models) [43] while controlling for age and gender covariates.

The dataset supporting the results of this article was deposited in the Sequence Read Archive (SRA) under BioProject accession code PRJNA650280. 


\section{Results}

\subsection{Severely Obese Subjects Show Significant Alterations in Blood Parameters Compared with Lean Controls}

The clinical and biochemical characteristics of the study cohort are shown in Table S1. Urea $(p$-value $\leq 0.001)$, glucose $(p$-value $\leq 0.001)$, and total cholesterol $(p$-value $\leq 0.05)$ levels were significantly higher in blood from obese subjects than in controls, whereas albumin levels were significantly lower in obese subjects ( $p$-value $\leq 0.05)$. The evaluation of the self-reported dietary habits highlighted important differences between $\mathrm{OB}$ and $\mathrm{CO}$ in terms of caloric intake (mean Kcal/day: 3.090 vs 1.618 , respectively, $p$-value $<0.0001$ ) and of eating habits. In fact, even if the percentages of main nutrients were similar (lipids: $28 \%$ vs $24.5 \%$; carbohydrates: 47.7 vs 49.5 ; proteins: $24.09 \%$ vs $25.9 \%$, in OB vs CO respectively), OB reported binge- and/or sweet-eating habits, 5-7 meals/day, and diets mostly containing simple and complex carbohydrates and lipids, whereas $\mathrm{CO}$ reported 3-4 meals/day containing preferentially complex carbohydrates and proteins.

\subsection{The Variability of the Bacterial Transcriptome Profile Is Higher in Obese than in Control Samples}

To investigate whether the microbiota transcriptome of obese $(\mathrm{OB})$ subjects differed from that of lean (CO) subjects, we aligned the reads to several sequence databases (see Materials and Methods) and extracted information at the gene, organism, subsystem, and pathway levels. The bacterial gene abundances revealed a significant difference between CO and OB samples (PERMANOVA, $\mathrm{R}^{2}=0.261$, $p$-value $=0.007$ ), as well as a considerable intragroup variability (Figure $1 \mathrm{~A})$. The heterogeneity of transcription regulation looked higher in obese microbiota than in control microbiota. The differential expression analysis revealed 55 significant differentially expressed genes (DEGs) between OB and $\mathrm{CO}$ samples (BH adjusted $p$-value $\leq 0.05$; $\log 2 \mathrm{FC} \geq|1|$ ) (Supplementary File 2 ). Figure $1 \mathrm{~B}$ shows the heat map of the variance stabilized counts of the DEGs. Hierarchical clustering revealed a separation between the two groups. Indeed, all but three CO samples clustered together and showed a homogeneous expression pattern. Although the OB samples were grouped together, they had two main inner clusters, as witnessed by the hierarchical branches and the color variation inside the heat map (Figure 1B). Interestingly, most genes (45/55) were overexpressed in OB vs CO samples. In terms of metabolic function, we found that $16 / 45$ (35.5\%) of these upregulated genes were involved in nucleotide metabolism, 9/45 (20\%) in carbohydrate metabolism, 9/45 (20\%) in the membrane transport system, and $4 / 45(8.9 \%)$ in amino acid metabolism, whereas the remaining $7 / 45(15.6 \%)$ were involved in bacterial defense mechanisms. The few down-expressed genes (10/55), highlighted by the clustering performed on the rows (Figure 1B), encoded mainly scaffolding and capsid proteins (Figure 1B).

A

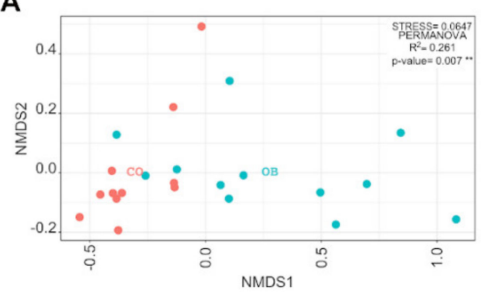

B

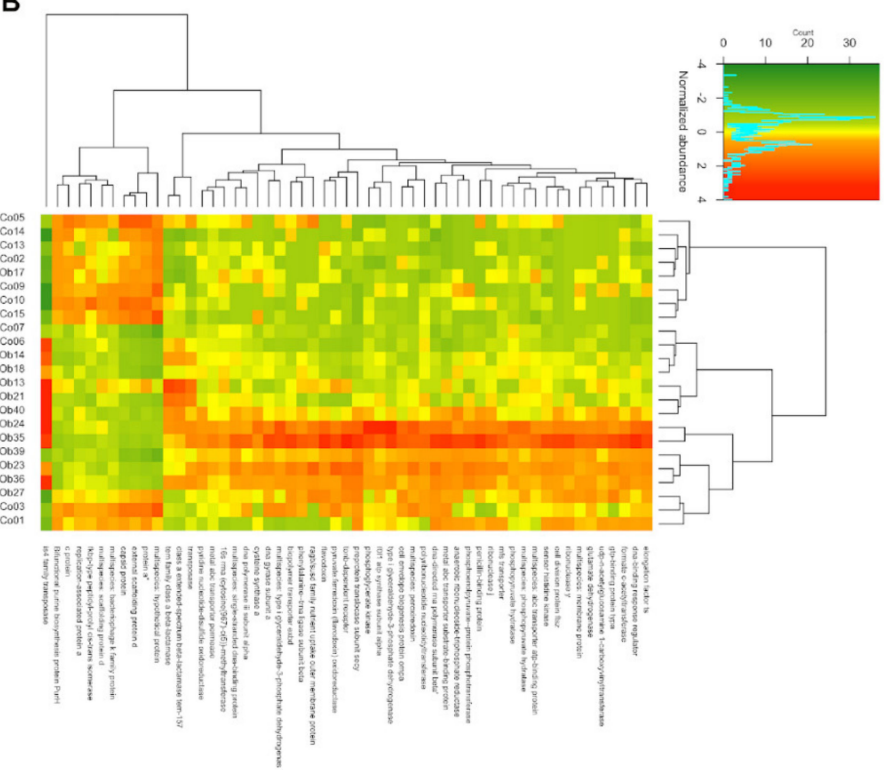


Figure 1. The bacterial transcriptome in Obese (OB) and Lean (CO) subjects. (A) Nonmetric multidimensional Scaling (NMDS) ordination of variance stabilized counts of bacterial RefSeq nonredundant proteins for $\mathrm{CO}(\mathrm{n}=11)$ and $\mathrm{OB}(\mathrm{n}=12)$ samples, compared using Bray-Curtis dissimilarity. The plot shows a higher heterogeneity in the case of obese samples and an overlapping region between the two groups, including four $\mathrm{CO}$ and three $\mathrm{OB}$ samples. The permutation analysis of variance (PERMANOVA) and corresponding R-squared and $p$-values, reported inside the plot, indicate a significant separation of the two classes. The Red and Cyan dots indicate CO and OB samples, respectively. (B) The heatmap-of-variance stabilized counts of bacterial RefSeq nonredundant proteins resulted in significant differences $(p<0.05$, Log2FC $>|1|)$ using DESeq2 differential analysis between the $\mathrm{OB}$ and $\mathrm{CO}$ groups. The Euclidean distance was calculated and the Ward's method was used for hierarchical clustering, both for rows and columns, as shown on top and right sides of the heatmap. Two distinct clusters are observable for the two classes, indicating that most of the functions were more highly expressed in the OB group. A third cluster (at the bottom) was composed of mixed samples from the two groups.

\subsection{Prevotella and Streptococcus Are Likely the Major Contributors to Some of the Altered Microbiota Metabolic Functions in Obese Subjects}

We evaluated taxa abundances by grouping the reads according to the organism annotations provided by the RefSeq sequences. The counts were normalized and used to perform the differential expression analysis. Figure 2A shows the nonmetric multidimensional scaling (NMDS) of samples using the normalized counts of all the organisms detected. The OB and CO cohorts were significantly separated (PERMANOVA, $\mathrm{R}^{2}=0.238, p$-value $=0.004$ ). The NMDS heterogeneity was higher in the CO samples than in those of Figure 1A, which suggests that several taxa contributed to fulfil the same functions. The high variability was comparable in OB samples with that of Figure 1A, apart from the two inner groups, which were no longer distinguishable. The heat map of the significantly different bacterial organisms (DEOs) (BH adjusted $p$-value $\leq 0.05$ and $\log 2 \mathrm{FC} \geq|1|$ ) is shown in Figure $2 \mathrm{~B}$. Interestingly, most bacterial species were more transcriptionally active in the $\mathrm{OB}$ than in the $\mathrm{CO}$ microbiomes. To investigate DEO activity, we realigned the reads to the RefSeq sequences of the organisms of interest (in this case, the DEO genera), as allowed by SAMSA2. We then analyzed the differential expression of these organism-specific genes. We obtained significant results in two cases: Prevotella (p_Bacteroidetes) and Streptococcus (p_Firmicutes). The specific functions assigned to Prevotella and Streptococcus showed significantly different results $(p$-value $<0.05)$ between OB and CO, as reported in Supplementary File 2. In order to verify whether the overall transcriptional activity of these two genera and their ratio were different between the two groups, we summed up all the counts matching their species obtained by grouping the reads according to the organism annotations and calculated the relative abundances at the genus (Prevotella and Streptococcus) and phylum (Bacteroidetes and Firmicutes) levels (Figure S1). Prevotella and Streptococcus abundances appeared different between $\mathrm{OB}$ and $\mathrm{CO}$ ( $p$-value: 0.07 and 0.012 , respectively), while the average of the abundance ratios calculated in all samples (Streptococcus/Prevotella) slightly changed between OB and $\mathrm{CO}(3.73$ vs $2.44, p$-value $=0.1)$. The average of the abundance ratios, summing the matches at the phylum level (Firmicutes/Bacteroidetes), was much higher in OB than in $\mathrm{CO}(5.4$ vs $2.38, p$-value $=0.06$ ) due to the higher average abundance of Firmicutes ( $p$-value $=0.0056$ ) (Figure S1). We also calculated the abundance ratios of Prevotella/Bacteroides, the most abundant genera within Bacteroidetes and, averaging the values of samples, we found that it was higher in OB than in CO samples (5.85 vs 2.42, $p$-value $=0.019)$. 
A

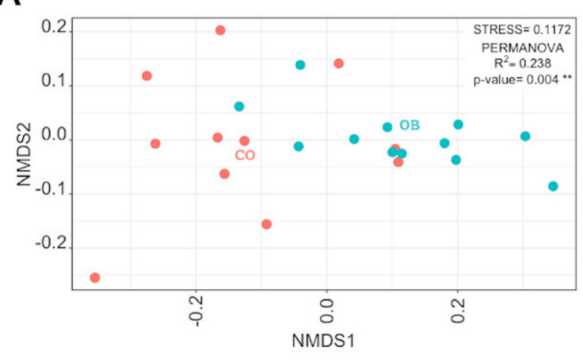

B

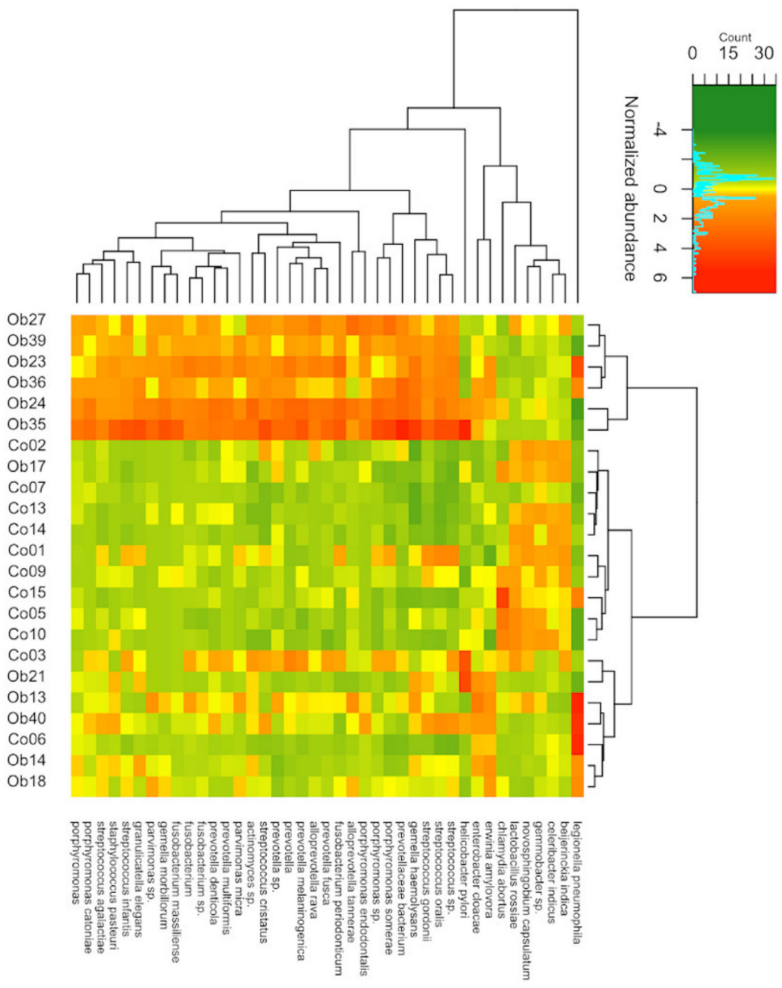

Figure 2. The bacterial organisms inferred from transcriptome data of Obese (OB) and Lean (CO) subjects. (A) Nonmetric multidimensional Scaling (NMDS) ordination of variance stabilized counts of bacterial organisms extracted from the alignment to the RefSeq nonredundant proteins database, for $\mathrm{CO}(\mathrm{n}=11)$ and $\mathrm{OB}(\mathrm{n}=12)$ samples, compared using Bray-Curtis dissimilarity. The plot shows a higher heterogeneity for both groups compared to the proteins plot (Figure 1A) and still shows an overlapping region between the two groups. The permutation analysis of variance (PERMANOVA) and corresponding R-squared and $p$-values, reported inside the plot, indicate a significant separation of the two classes. The Red and Cyan dots indicate $\mathrm{CO}$ and OB samples, respectively. (B) Heatmap of variance stabilized counts of bacterial organisms extracted from the alignment to the RefSeq nonredundant proteins database, resulted in significant differences ( $p$-value $<0.05$, Log2FC $>|1|)$ using DESeq2 differential analysis between $\mathrm{OB}$ and $\mathrm{CO}$ groups. The Euclidean distance was calculated, and the Ward's method was used for hierarchical clustering, both for rows and columns, as shown on the top and right sides of the heatmap. A distinct cluster for a subset of obese samples is observable on the top.

\subsection{Taxa Abundance May Not Correspond to Transcriptional Activity}

We compared the organism abundances inferred from the alignment to the RefSeq protein sequences to those that we recently obtained using $16 \mathrm{~S}$ rRNA sequencing in a different cohort of obese and lean control subjects, as well as in 13 samples that were common to both studies $(6 \mathrm{CO}$, $7 \mathrm{OB}$ ) [24] (Figure 3A,B). As shown in Figure 3, the microbiome composition at phylum level, obtained from the taxonomic assignment of $16 \mathrm{~S}$ rRNA (Figure $3 \mathrm{~A}$ ) versus that inferred from mRNA analysis performed in the present study (Figure 3B), revealed that the Firmicutes and Proteobacteria phyla were, respectively, less and more abundant in terms of $16 \mathrm{~S}$ counts, but showed an opposite trend for the transcriptional activity. In addition, the Actinobacteria phylum was less abundant and also significantly less transcriptionally active in $\mathrm{OB}$ than in $\mathrm{CO}$ (Figure 3A,B). Similar results were obtained for both, considering the two whole cohorts of individuals and the subgroup of 13 subjects common to both experiments (see right smaller plots in A and B panels of Figure 3). 
A
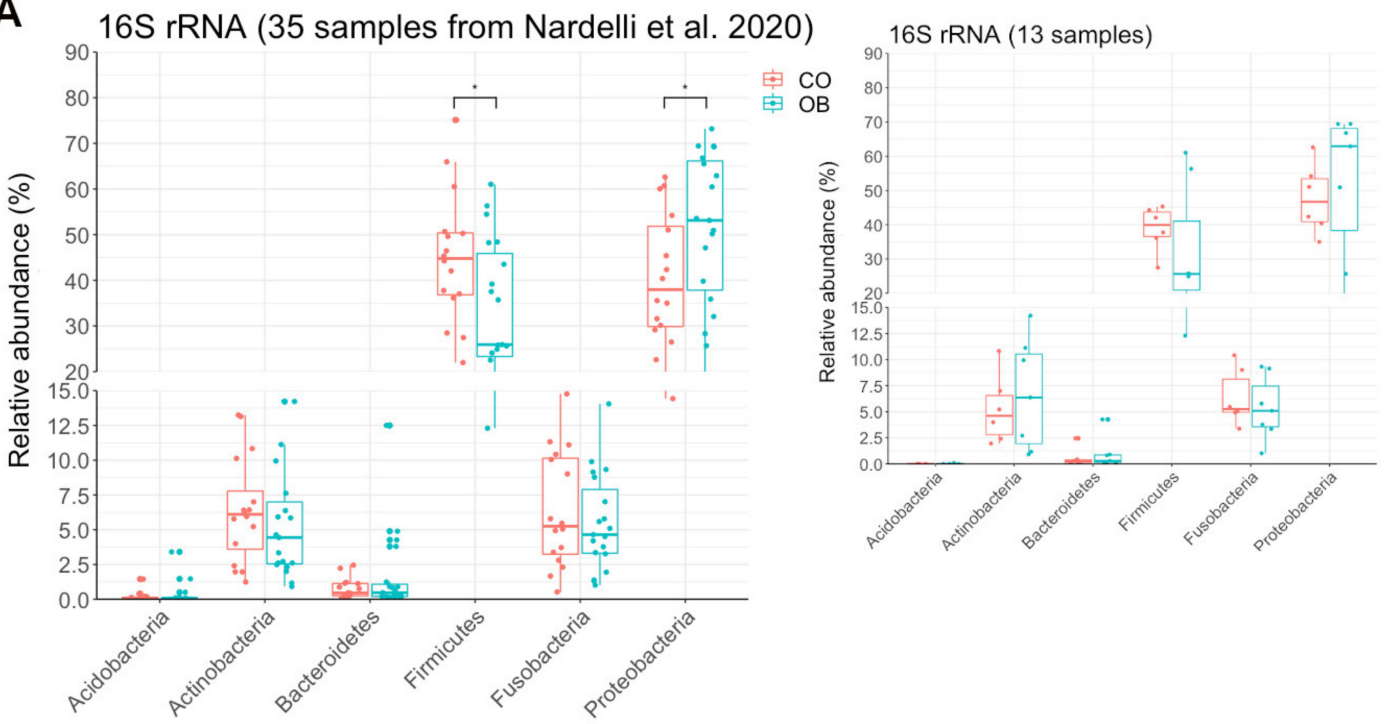

B
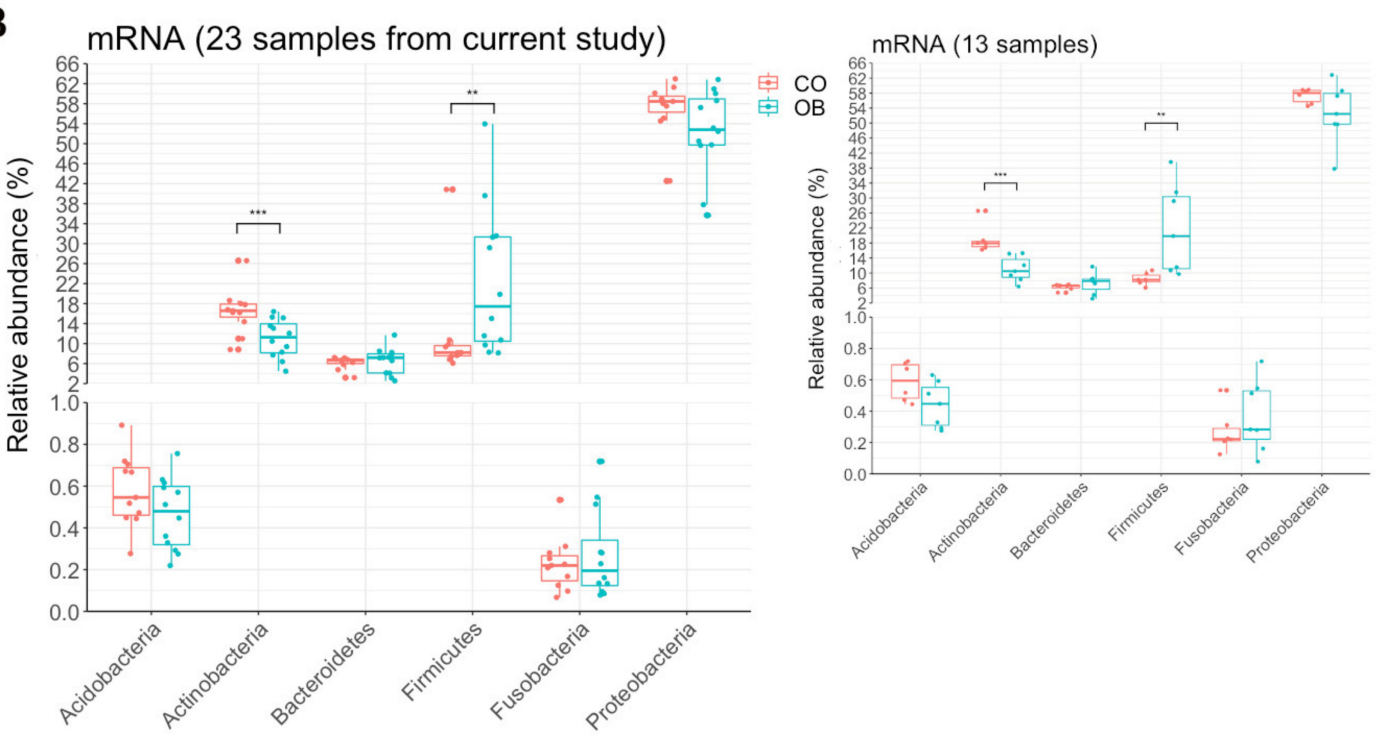

Figure 3. Phyla abundances in Obese (OB) and Lean (CO) subjects obtained by $16 \mathrm{~S}$ rRNA sequencing and inferred from transcriptomic data. The boxplots show the relative abundances of phyla obtained through 16S shotgun sequencing performed in our previous study (Nardelli et al., 2020) (A) and inferred by the mRNA gene abundances in the current study (B), containing 35 and 23 samples, respectively. Among these, 13 patients (6 CO and $7 \mathrm{OB}$ ) were analyzed in terms of both 16S rRNA and transcriptomics and are shown by the right smaller plots. The middle line in the boxes represents the median, lower box bounds represent the first quartile, and upper box bounds represent the third quartile. Whiskers represent the $95 \%$ confidence interval of the mean. The significance of distribution differences was calculated by applying the Kruskal-Wallis test and Benjamini-Hochberg $p$-value adjustment $\left({ }^{*} p<0.05\right.$; ** $\left.p<0.01 ;{ }^{* *} p<0.005\right)$. The Red and Cyan boxplots indicate control and obese samples, respectively.

\subsection{Carbohydrate, Amino-Acid, and Nucleotide Metabolism Are Impaired in Obese Microbiota}

We exploited SEED subsystem hierarchies to group genes into functional categories and, thus, to investigate the overall activity of microbiota organisms in the OB and CO individuals. Briefly, SEED is a categorization system, which organizes functional categories of homologous genes into a hierarchy with five levels of resolution. Homologous genes are known to share the same function. The differential expression analysis was performed for each level of grouping, from 1 (the most generic) to 4 (individual functions). Level 4 (L4) and 3 (L3) differentially expressed terms are reported in Supplementary File 3. As expected, many L4 terms were redundant with the RefSeq genes. Consequently, we focused on L3, 
which provides an upper categorization, and the significantly upregulated terms were further grouped into level 2 and level 1 (Figure 4) to obtain a better idea of the overall functionality. The weight of the alterations and the quantity of terms involved in particular categories is reflected in the size of the slices of the pie chart, which are proportional to the Log2FC values. The figure shows that the most dominant and active functionalities in the duodenum gut microbiota of obese subjects were the metabolism of carbohydrates (16.42\%), DNA and RNA (14.09\%), and amino acids and derivatives $(9.63 \%)$. Other upregulated terms were the Clustering-based subsystems group (terms with unknown function) (10.02\%); the Virulence, Disease, and Defense mechanisms (9.03\%); Membrane transport $(8.92 \%)$; and Cell wall and capsule $(8.57 \%)$. The microbial functional profiles were further summarized as MetaCyc pathways. In order to identify pathways specifically associated with obesity and to exclude confounding factors (covariates), such as gender and age, we performed a multivariate analysis using the generalized linear models in MaAsLin2 (Multivariate Association with Linear Models). Nine metabolic pathways were predicted to be significantly associated with obesity (Figure 5), all of which were related to the metabolism of nucleotides and amino acids.

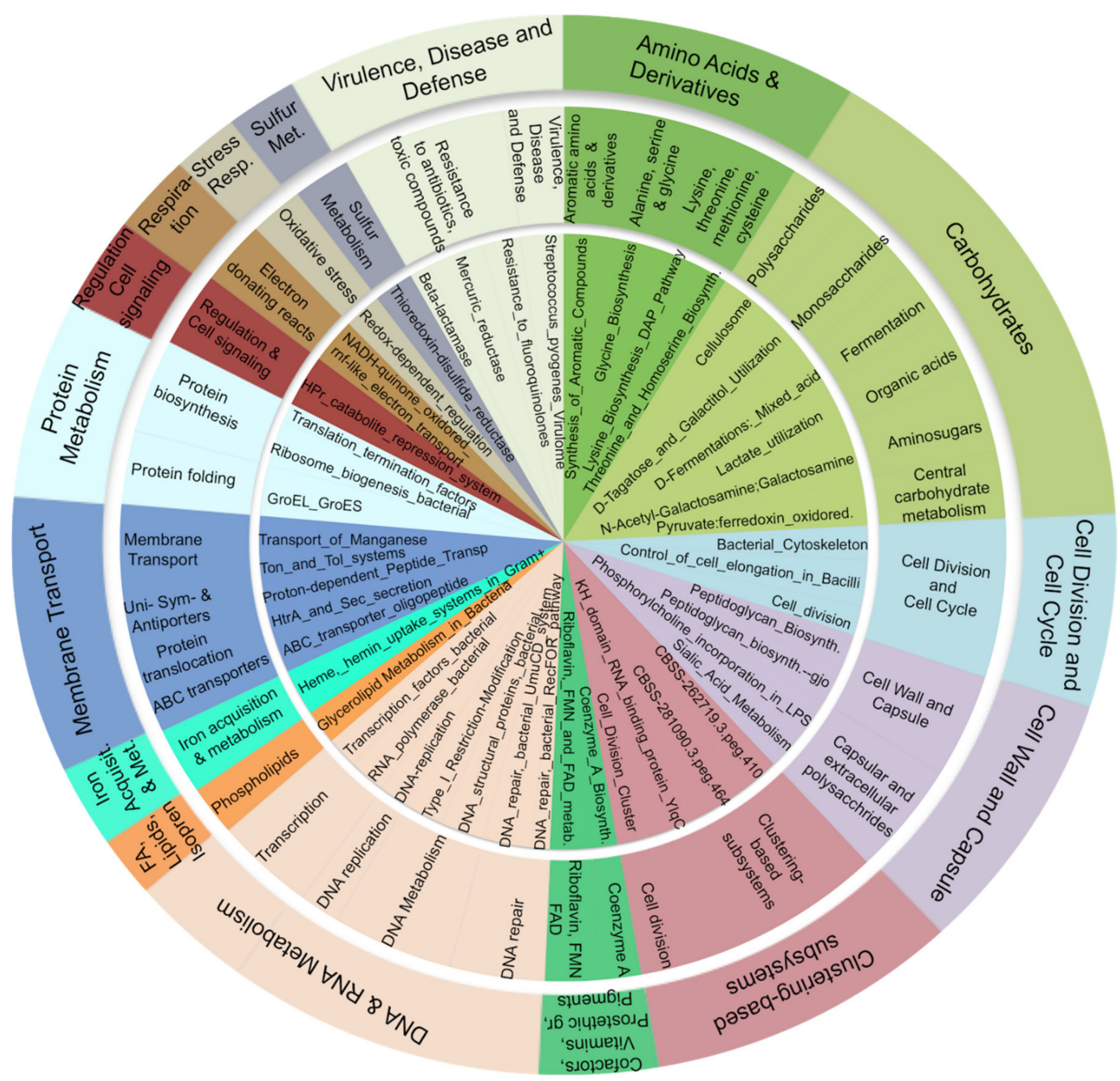

Figure 4. SEED subsystem hierarchy. The level 3 terms of SEED subsystem database (shown in the inner circle of the figure) resulted significant up-expressed genes $(p<0.05$, $\log 2 \mathrm{FC}>1)$ between Lean $(\mathrm{CO}, \mathrm{n}=11)$ and Obese $(\mathrm{OB}, \mathrm{n}=12)$ samples using DESeq2 differential abundance analysis. Terms were then grouped according to their belonging to higher levels 2 (middle circle) and 1 (outer circle). The slices size is proportional to the Log2FC values. The figure shows that the most altered functions concern the Amino acids, Carbohydrates, and Nucleic acids metabolism; the Clustering-based subsystem; the Virulence, Disease, and Defense mechanisms; and the Membrane transport. 


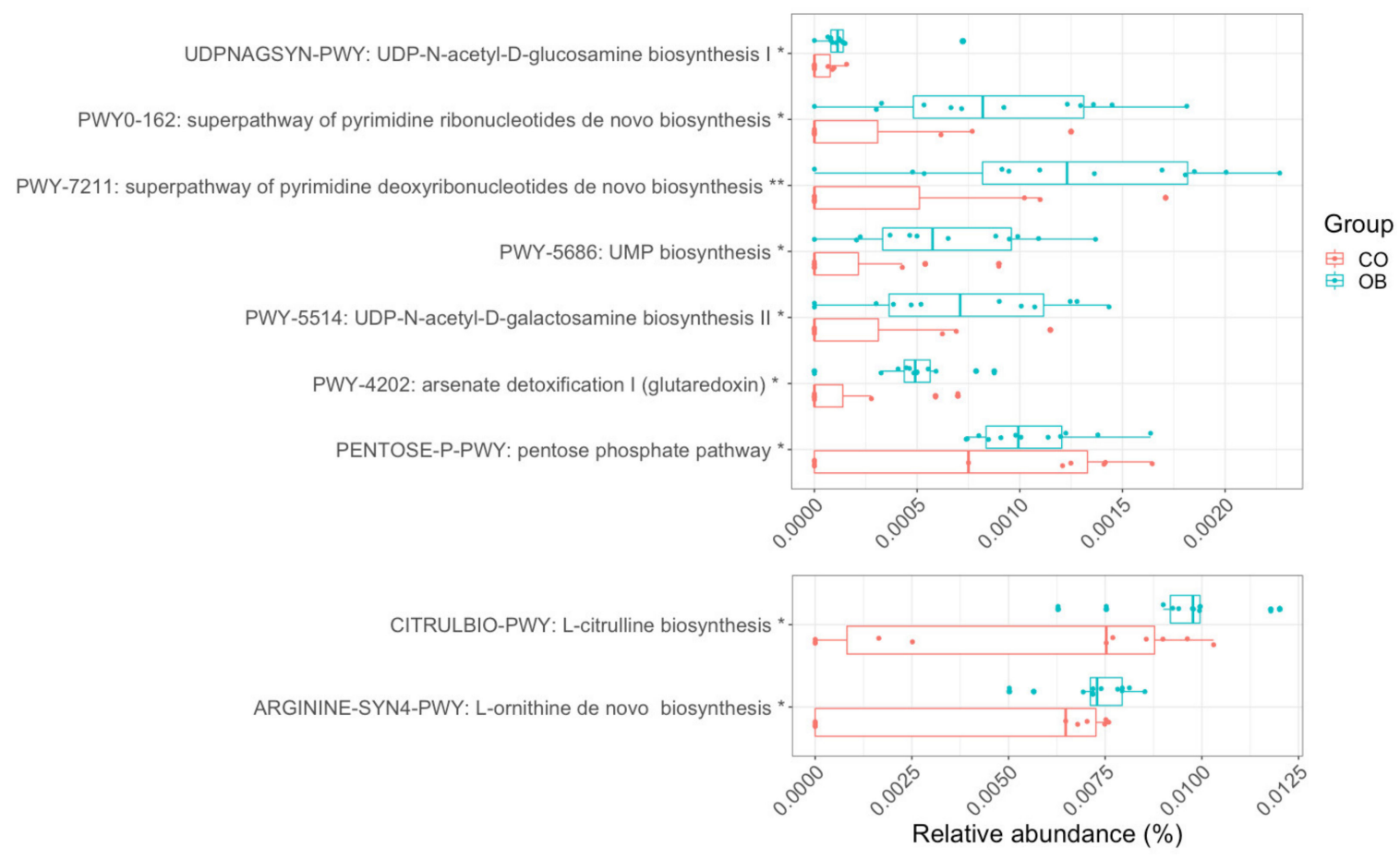

Figure 5. MetaCyc microbial processes associated with obesity $(\mathrm{q}<0.1)$ detected using the MaAsLin2-generalized linear model. The significance was calculated after adjusting for age and gender effects. The $\mathrm{x}$-axis shows the relative abundances. Boxplots were made with $\mathrm{R}$ function boxplot with default settings (whiskers extend 1.5-times the interquartile range). $\left({ }^{*}: 0.05<\mathrm{q}<0.1\right.$ : $* *: 0.01<\mathrm{q}<0.05)$. Red and Cyan boxplots indicate Lean $(\mathrm{CO}, \mathrm{n}=11)$ and Obese $(\mathrm{OB}, \mathrm{n}=12)$ samples, respectively.

\subsection{Differentially Expressed Host Genes Share the Same Metabolic Pathways as Microbial Genes}

We analyzed the human mRNA from the total RNAs of the duodenal niche to look for convergences between the microbiota and its host. The differential abundance analysis of human genes revealed 40 differentially expressed transcripts between $\mathrm{OB}$ and $\mathrm{CO}$ samples, of which 15 were upregulated and 25 downregulated (BH adjusted $p$-value $\leq 0.05$ and Log2FC $\geq|1|$ ) (Supplementary File 4). Based on KEGG and Gene Ontology annotations (Supplementary File 4), we grouped the genes according to their overall functional implication and found that they were mostly involved in Immunity/Inflammation/Apoptosis; the Structure and Function of Nucleic Acids; and Cellular components/functions: Membrane structure, Cytoskeleton, and Metabolism (Lipids, Carbohydrates, Proteins) (Table 1). 
Table 1. Significantly up-expressed and down-expressed human genes in Obese (OB) vs Lean (CO) and their putative role (based on KEGG and GOTERM databases).

\begin{tabular}{|c|c|c|c|c|c|}
\hline & Transcripts & Localization & $\log 2 \mathrm{FC}$ & Adjusted $p$-Value & Putative Role * \\
\hline BCL2 & Apoptosis regulator & Mitochondrial membrane & -1.82 & 0.004 & \multirow{9}{*}{$\begin{array}{c}\text { Immunity/Inflammation/ } \\
\text { Apoptosis }\end{array}$} \\
\hline TNFRSF13B & TNF receptor superfamily member $13 \mathrm{~B}$ & Plasma membrane & -1.44 & 0.031 & \\
\hline CSF1R & Colony stimulating factor 1 receptor & Membrane & -1.62 & 0.037 & \\
\hline ACP5 & Acid phosphatase 5, tartrate resistant & Lysosome & -1.06 & 0.027 & \\
\hline IGLL5 & Immunoglobulin lambda like polypeptide 5 & Extracellular localization & -1.75 & 0.027 & \\
\hline ANXA1 & Annexin 1 & Different cellular localization & -2.12 & 0.039 & \\
\hline ERBB3 & Erb-b2 receptor tyrosine kinase 3 & Plasma membrane & -24.56 & $<0.0001$ & \\
\hline NGEF & Neuronal guanine nucleotide exchange factor & Membrane & 3.66 & 0.025 & \\
\hline $\mathrm{P} 4 \mathrm{HB}$ & Prolyl 4-hydroxylase subunit beta & Cytoplasm & 21.74 & $<0.0001$ & \\
\hline PRMT5 & Protein arginine methyltransferase 5 & Nucleus and cytoplasm & -7.43 & 0.0001 & \multirow{8}{*}{$\begin{array}{l}\text { Nucleic acids structure } \\
\text { and functions }\end{array}$} \\
\hline TSEN34 & tRNA splicing endonuclease subunit 34 & Nucleus & -5.8 & 0.031 & \\
\hline EIF3CL & $\begin{array}{l}\text { Eukaryotic translation initiation factor } 3 \text { subunit } \\
\text { C-like }\end{array}$ & Cytoplasm & 21.82 & $<0.0001$ & \\
\hline ELF2 & E74 like ETS transcription factor 2 & Nucleus and cytoplasm & 6.94 & 0.008 & \\
\hline WBP2 & WW domain binding protein 2 & Nucleus and cytoplasm & 2.88 & 0.0022 & \\
\hline ZNF195 & Zinc finger protein 195 & Nucleus & 3.21 & 0.033 & \\
\hline SRRT & Serrate, RNA effector molecule & Nucleus & 6.18 & $<0.0001$ & \\
\hline PDE4A & Phosphodiesterase 4 & Cytoplasm & -1.22 & 0.015 & \\
\hline ANXA2 & Annexin 2 & Different cellular localization & 1.69 & 0.012 & \multirow{16}{*}{$\begin{array}{c}\text { Cellular } \\
\text { components/functions: } \\
\text { membrane structure, } \\
\text { cytoskeleton }\end{array}$} \\
\hline IQGAP1 & IQ motif containing GTPase activating protein 1 & Plasma membrane & -1.73 & 0.008 & \\
\hline WDR1 & WD repeat domain 1 & Different cellular localization & -24.6 & $<0.0001$ & \\
\hline ANXA1 & Annexin 1 & Nucleus & -2.12 & 0.039 & \\
\hline BANF1 & Barrier to autointegration factor 1 & Different cellular localization & -1.15 & 0.041 & \\
\hline CDR1 & Cerebellar degeneration related protein 1 & Nucleus & -2.53 & 0.012 & \\
\hline FAM192A & Family with sequence similarity 192 member A & Plasma membrane & -5.59 & 0.014 & \\
\hline PARD3 & Par-3 family cell polarity regulator & Cytoplasm and membrane & -8.32 & 0.023 & \\
\hline PLEKHB1 & Pleckstrin homology domain containing B1 & Plasma membrane & -1.23 & 0.012 & \\
\hline RGMA & Repulsive guidance molecule family member a & Cytoplasm & -4.68 & 0.037 & \\
\hline CLASP1 & Cytoplasmic linker associated protein 1 & Plasma membrane & 3.80 & 0.014 & \\
\hline CD82 & CD82 molecule & Membrane & 5.78 & 0.041 & \\
\hline DAG1 & Dystroglycan 1 & Membrane & 4.47 & 0.009 & \\
\hline ILDR1 & Immunoglobulin like domain containing receptor 1 & Membrane & 6.24 & 0.027 & \\
\hline LMNA & Lamin $\mathrm{A} / \mathrm{C}$ & Nucleus & -9.63 & 0.028 & \\
\hline CASC4 & Cancer susceptibility candidate 4 & Membrane & 7.62 & $<0.0001$ & \\
\hline
\end{tabular}


Table 1. Cont.

\begin{tabular}{|c|c|c|c|c|c|}
\hline & Transcripts & Localization & $\log 2 \mathrm{FC}$ & Adjusted $p$-Value & Putative Role * \\
\hline MME & membrane metalloendopeptidase & Membrane & $20.61(\mathrm{P})$ & $<0.0001$ & \multirow{11}{*}{$\begin{array}{c}\text { Metabolism: lipid (L) } \\
\text { carbohydrates (C), } \\
\text { protein (P) }\end{array}$} \\
\hline PPT1 & palmitoyl-protein thioesterase 1 & Lysosome & $-1.37(\mathrm{~L})$ & 0.014 & \\
\hline MGEA5 & Meningioma expressed antigen 5 (hyaluronidase) & Nucleus & $\begin{array}{c}-24.08 \\
(C)\end{array}$ & $<0.0001$ & \\
\hline RAP1GDS1 & Rap1 GTPase-GDP dissociation stimulator 1 & Cytoplasm & $-4.02(\mathrm{~L})$ & 0.045 & \\
\hline LMNA & Lamin $\mathrm{A} / \mathrm{C}$ & Nucleus & $\begin{array}{l}-9.63 \\
(\mathrm{~L}+\mathrm{C})\end{array}$ & 0.028 & \\
\hline PGA3 & Pepsinogen 3 , group I & Different cellular localization & $-5.92(\mathrm{P})$ & 0.031 & \\
\hline CALM2 & Calmodulin 2 & Different cellular localization & $\begin{array}{c}10.91 \\
(\mathrm{~L}+\mathrm{C})\end{array}$ & $<0.0001$ & \\
\hline PIK3C2B & $\begin{array}{c}\text { Phosphatidylinositol-4-phosphate 3-kinase catalytic } \\
\text { subunit type } 2 \text { beta }\end{array}$ & Cytosol & $-7.39(\mathrm{~L})$ & 0.027 & \\
\hline ADH1B & Alcohol dehydrogenase 1B & Different cellular localization & $\begin{array}{c}3.41 \\
(\mathrm{~L}+\mathrm{C})\end{array}$ & 0.005 & \\
\hline ANXA2 & Annexin 2 & Different cellular localization & $\begin{array}{c}1.69 \\
(\mathrm{~L}+\mathrm{C})\end{array}$ & 0.012 & \\
\hline BCL2 & Apoptosis regulator & Mitochondrial membrane & $-1.82(\mathrm{~L})$ & 0.004 & \\
\hline
\end{tabular}

* Based on KEGG and GEOTERM database. 
Interestingly, microbial and host genes intersected at the level of Energy, Carbohydrate, and Amino Acid Metabolism (Figure 6). Other intersections occurred at the levels of Lipid and Nucleotide metabolism, in which differentially expressed host genes played a role.

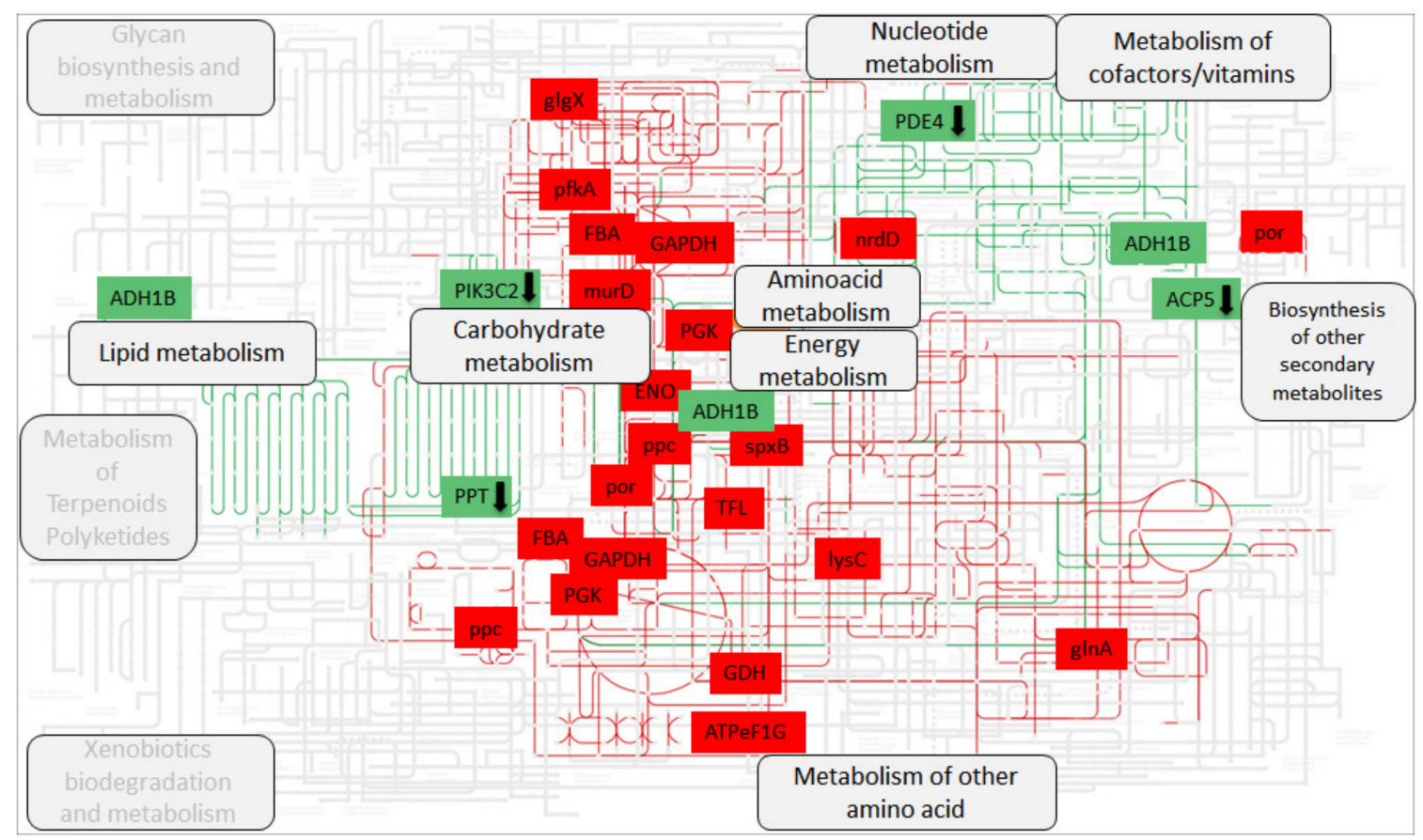

Figure 6. Human and microbial genes mapped into metabolism roadmap. The figure shows the metabolic pathways involving the differentially expressed genes between Obese (OB) and Lean (CO). The SEED subsystem L4 (Supplementary File 3) and human genes differentially expressed between the two groups (Table 1 and Supplementary File 4) were converted into KEGG orthologs Ids and mapped to the human metabolism using iPath3. The pathways related to the matched orthologs are highlighted to identify convergent paths between host and bacteria, colored in green and red, respectively. The genes which found one or multiple matches are also shown and highlighted by the corresponding colors. The genes reported in the figure were all up-expressed in OB vs CO, except a few which were down-expressed, indicated by an arrow in the box $(n=4)$. Whole gene names and corresponding EC numbers are reported below in "Abbreviations" section.

\section{Discussion}

Although the gut microbiota composition is intimately associated with human health, to our knowledge, the functional activities driven by specific microbial configurations and transcription in severe obesity have not yet been elucidated. Here, we report the results of a pilot study of the human duodenal metatranscriptome in a small cohort of severely obese and lean control subjects from Southern Italy. We describe the metatranscriptomics profile associated with obesity, which includes transcripts, the contributing bacterial organisms, and the specialized pathways differentially expressed between the two groups of participants; a comparison between bacterial composition and transcriptome-related data of the human duodenal microbiome; and, finally, the putative convergence on key metabolic pathways between host and microbial transcripts, which might be associated with the obesity-associated phenotype.

\subsection{Microbiome Composition by $16 S$ rRNA Compared to Microbiome Inferred by Transcriptome}

Recent experimental evidence has highlighted that not all the microbes detected in a human niche are equally active. Therefore, the functional activity of the microbiota should be evaluated in addition to its composition [26,27]. Using 16S rRNA gene sequencing, we recently studied the duodenal microbiome of an obese cohort of subjects from Southern Italy [24]. Here, we describe 
the duodenal metatranscriptome in another cohort of severely obese and lean control subjects from Southern Italy. First, we observed a high heterogeneity of the gut microbiome gene expression between and within the two groups of $\mathrm{OB}$ and $\mathrm{CO}$ subjects. Although interindividual variability is a well-known aspect of microbiota due to the many contributing aspects, this result suggests a higher microbial variability in obese subjects, which could be due to the various obesity driving factors. These results are in agreement with the high interindividual variability previously observed in the composition of human gut microbiome by $16 \mathrm{~S}$ rRNA sequencing and mainly attributed to environmental factors and BMI [25]. Despite this, the microbial expression profiles differed significantly between OB and CO subjects. This finding indicates that a typical functional activity is associated with obesity. Furthermore, the comparison between the transcriptional activity of specific taxa inferred by transcriptome analysis, as well as their abundance previously detected by $16 \mathrm{~S}$ rRNA gene sequencing [24], suggested that they did not show the same behavior. In detail, in the $16 \mathrm{~S}$ rRNA analysis, Firmicutes and Proteobacteria phyla were less and more abundant in OB than in CO samples, respectively [24], and had opposite trends in the case of mRNA expression abundances. Our data support the previously observed data in agreement with species abundance and transcriptional activity in human microbiomes from fecal samples of 341 elderly men [27]. This discrepancy was not unexpected because, under steady-state conditions, transcription is a highly variable process, even among cells of the same species that may be influenced by many factors, including nutrient availability [44]. To support the nutrient influence on bacterial gene expression, D'Souza and Kost [45] experimentally demonstrated that a loss of metabolic functions was likely when the corresponding metabolites could be derived from the environment. In particular, populations of Escherichia coli that evolved in amino acid-replete environments rapidly lost the ability to autonomously produce several amino acids, which was beneficial when amino acids were present in the environment [45]. Essentially, we found a significantly higher functionally active Firmicutes and a higher Firmicutes/Bacteroidetes ratio in OB than in CO samples. These findings coincide with previous findings obtained in fecal samples from obese mice [46] and obese patients [47].

\subsection{An Increased Prevotella/Bacteroides Activity Ratio Is Associated with Obesity}

Among the Bacteroidetes, the Bacteroides and Prevotella genera are the most abundant, usually inhabiting the gut and oral cavity, respectively. When present in the same niche, one or the other genus predominates, acting as an antagonist [48]. They have also been suggested as biomarkers of diet and lifestyle [49]. Here, we found that the Prevotella genus was more active than the Bacteroides genus in the duodenum of obese patients compared to lean subjects. As known, Prevotella and Bacteroides characterize the two most frequently found "enterotypes" in Western and non-Western subjects, respectively, with the Prevotella-driven enterotype represented at high values in individuals consuming a carbohydrate-based diet [50]. An abundance of the Prevotella genus has been also associated with the Mediterranean diet [51], and an increased Prevotella/Bacteroides ratio has been associated with higher BMI and a higher susceptibility of obese subjects to regain weight in the case of a low dietary fiber intake and impaired glucose metabolism [52]. Our OB cohort self-reported a Mediterranean albeit hypercaloric diet (mean Kcal/day: 3.090), which contained preferentially simple and complex carbohydrates and lipids. Nevertheless, the role of diet in tipping the outcome of the competition between Bacteroides and Prevotella in the duodenum of our obese subjects remains to be ascertained.

\subsection{The Bacterial Functional Profile in Duodenum Differs between Obese and Lean Control Subjects}

Bacterial transcriptome profiling revealed differences in terms of gene expression between the severely obese and lean subjects. Below, we discuss the most relevant differences associated with obesity.

\subsubsection{Carbohydrate Metabolism}

Using alignment to the SEED subsystem database, we found that several enzymes involved in the Embden-Meyerhoff-Parnas (EMP) pathway (namely aldolase, enolase, pyruvate kinase, 
phosphoglycerate kinase, pyruvate formate-lyase, and pyruvate-ferredoxin oxidoreductase) were upregulated in obese subjects. In agreement, when we grouped gene families in MetaCyc pathways, we found that the Pentose-phosphate-pathway was one of the metabolic networks associated with OB duodenal microbiomes. Bacterial carbohydrate metabolism, through glycolysis and succinate production from fumarate (first oxidative branch), as well as acetate and formate from pyruvate (second oxidative branch), are involved in the central energy and carbon metabolism of Prevotella spp. [53]. Despite the competitive relationship between Prevotella and Bacteroides, these two bacteria share many of the above described enzyme activities. However, unlike Bacteroides, Prevotella does not produce propionate. The latter feature could modify the abundance of some short-chain fatty acids to be used by the host's colonocytes and could thus affect human host physiology as previously described [53]. Further, De Angelis et al. [54], using a multi-omics approach, recently studied how diet influences the functions of the human intestinal microbiome in fecal samples from volunteers with different (omnivorous, vegetarians, and vegans) dietary habits. The authors reported that phylogenetic composition was not useful in discriminating different dietary habits, but several genera were associated to the intake of specific dietary components [54]. In detail, the intestinal microbiome of individuals with vegetable-rich diets showed significant differences in relative abundances of several genes, including those involved in carbohydrate transport and metabolism [54]. In addition, higher diversity at the subgenus levels of human fecal Prevotella and Bacteroides have also previously been associated with specific dietary patterns [55]. Based on the above, we could hypothesize that the upregulation of carbohydrate metabolism found in the duodenal microbiome of our obese cohort may be diet-driven.

\subsubsection{Cell Wall}

UDP-N-acetylgalactosamine biosynthesis is another MetaCyc pathway which was associated with OB samples. N-acetylgalactosamine is a carbohydrate molecule that, in its activated form, participates in the constitution of polymer structures, together with lipopolysaccharides, peptidoglycans, and glycans $\mathrm{N}$ and $\mathrm{O}$, which are linked to proteins [56]. Glycosylated proteins play important roles in the cell membrane in vivo. In fact, they are often involved in cell-cell adhesion, cytoskeleton regulation, and immune recognition [56]. Lipopolysaccharide (LPS), a glycoconjugate present in the outer membrane (OM) of Gram-negative bacteria, is an important immunomodulatory molecule, contributes significantly to the structural integrity of the OM, and helps to shield the OM from antibiotics and other environmental attacks [57]. Plasma levels of LPS increased in obese animal models (ob/ob mice) as compared to lean mice in association with gut microbiota change (fewer Bacteroidetes and more Firmicutes), decreased tight junctions, and increased gut permeability [46,58]. In our study, we found the "Phosphorylcholine (ChoP) incorporation in lipopolysaccharide (LPS)" was up-expressed according to the SEED subsystem database. Interestingly, it was previously demonstrated that the attachment of the small molecule ChoP to the LPS, covering the bacterial surface, evaded immune responses during colonization in its human host [59]. We hypothesize that such mechanism could be associated with the LPS increase previously described in obesity [60].

\subsubsection{Defense Mechanisms: The Arsenate Detoxification Pathway}

We found that the bacterial arsenate detoxification pathway was associated with obesity. Arsenic is ubiquitous in the environment and in the form of arsenate, which toxic to all living organisms. Thus, microorganisms have evolved various mechanisms to detoxify arsenate [61,62]. Interestingly, arsenate was reported to interfere with glucose, energy, and phosphate metabolism [61].

\subsubsection{Amino Acids and Derivatives}

Gut microbiota utilizes undigested proteins, together with endogenously synthesized proteins, to generate amino acids such as arginine, which is converted via citrulline into L-ornithine, a precursor for polyamine production whose increased levels negatively affect gut health [63]. Interestingly, among the significantly up-expressed MetaCyc pathways in our obese cohort were those of L-ornithine 
and L-citrulline biosynthesis, which are both alternative arginine amino acid metabolic pathways that can lead to proinflammatory or immunoregulatory effects [63].

\subsubsection{Nucleic Acids}

The de novo pyrimidine biosynthesis pathway predominates during intensive growth in mammals and most other organisms [64]. The latter pathway enables these organisms to synthesize nucleotides from small metabolites. The de novo pyrimidine biosynthesis pathway is also essential for the proliferation of many pathogens [64]. We found the de novo pyrimidine biosynthesis pathway was up-expressed in our cohort. Thus, we may speculate that it could contribute to obesity-associated dysbiosis.

\subsection{The Human Tanscriptional Profile in the Duodenum Differs between Obese and Lean Subjects}

Most of the human transcripts in the duodenum of our OB patients were down-expressed compared to lean subjects. In particular, the down-expression of ErbB3 and of CSF1R genes, observed in our obese cohort, was previously described to alter intestinal cells differentiation [65,66]. Indeed, ErbB3 is expressed in the small intestine, and its signaling restricts the number of Paneth cells (PCs). This effect requires PI3K signaling [65], which also was down-expressed in our obese subjects. Accordingly, the intestinal epithelium of ErbB3 (-/-) mice was sensitized to inflammation and the PC expansion was altered, thereby decreasing the release of antimicrobial peptides [65]. Regarding CSF1R, this gene is expressed in macrophages, which are associated with the crypt epithelium and involved in the maintenance of the intestinal stem cell niche and PC differentiation [66]. As known, Paneth cells release antimicrobial lysozyme and $\alpha$-defensins, together with other factors essential for the maintenance of intestinal stem cells [67]. Globally, defects in PCs or in their production of antimicrobial factors can result in intestinal dysbiosis [66]. Based on the above assumptions, we may hypothesize that the down-expression of ErbB3 and of CSF1R genes, observed in the duodenum of our obese subjects, could be associated with gut epithelial dysfunction and dysbiosis. Another down-expressed gene in our obese group was AnxA1. The latter participates in cell differentiation, membrane transport, motility, signal transduction, inflammation, and apoptosis [68]. Interestingly, the relationship between AnxA1, obesity, and obese-associated metabolic diseases has been well documented due to the role of AnxA1 in the treatment of type-2 diabetes [69-72]. In fact, AnxA1-null mice fed a high-fat diet were more obese and had larger adipocytes than wild-type mice fed a high-fat diet, whereas recombinant human AnxA1 treatment reduced body weight, fat mass, and liver steatosis [72]. Intestinal epithelial integrity and cell-to-cell adhesion are essential to ensure gut function and homeostasis. In fact, tight junctions control paracellular permeability (gate functions) [73]. The other deregulated transcripts in our obese cohort were IQGAP1, AnxA2, calmodulin, and WDR1. Interestingly, all of them could be involved putatively in intestinal cell membrane composition, gate function, and inflammatory bowel diseases [68]. In detail, the IQ motif containing GTPase-activating protein1 (IQGAP1), a member of a family of scaffolding proteins, is involved in the differential recruitment of claudins to nascent tight junctions [74]. Among the 300 interacting proteins documented for IQGAP1, AnxA2 and calmodulin were both up-expressed in duodenum of obese subjects. In particular, calmodulin competes for binding to IQGAP1 both in vitro and in a normal cellular milieu [75]. Further, WD-repeat protein1 (WDR1), also known as "actin-interacting protein 1," plays a significant role in actin cytoskeletal remodeling by promoting the assembly of apical cell-cell junctions [76]. Lastly, the down-expression of Lamin in OB duodenum could likely contribute to the deregulation of gene expression associated with obesity. In fact, Lamins $\mathrm{A} / \mathrm{C}$ are nuclear proteins that polymerize to form the nuclear lamina, which is a filamentous network located just below the inner nuclear membrane that assists in maintaining nuclear membrane integrity and controlling gene expression [77]. Accordingly, defective A-type lamins have been previously described in conditions such as regional adiposity, type-2 diabetes, and aging [78].

Globally, the human transcriptome profile of duodenum found in our obese cohort could likely be responsible for the dysfunction of the intestinal epithelium composition, intestinal permeability 
function, and inflammation, all of which may be associated with the obese-associated dysbiosis and may alter key metabolic pathways.

\subsection{Putative Convergence between Host and Microbial Transcriptomes}

Intriguingly, among the human obese-associated transcripts, the up-expressed Alcohol dehydrogenase 1B (ADH1B) and the down-expressed Palmitoyl-protein thioesterase 1 (PPT1) and Phoshatidylinositol-4-phospate 3-kinase subunit beta (PIK3C2B) were all involved in the metabolic pathways to which bacteria also contribute, and may be potentially be associated with the obese phenotype. In fact, the expression of ADH1B was found altered in the adipose tissue of obese subjects and was associated with BMI and waist circumference [79]. A deficiency of PPT1 activity and altered cholesterol metabolism have been reported in brain tissue in association with local neuroinflammation [80], as well as in Sertoli cells in association with male subfertility, abnormal lysosome accumulation, and increased cholesterol levels [81]. In agreement with the above data, the down-expression of PPT1 observed in our OB subjects was associated with significantly increased serum cholesterol levels. Finally, PIK3C2B, which we found to be down-expressed, is an enzyme ubiquitously expressed in human tissues and a controller of endosome trafficking, whose inactivation alters insulin receptor trafficking [82].

Our pilot study presents some limits. First, we studied a small cohort. However, all subjects were from the Campania Region of Southern Italy and self-reported similar dietary habits, thereby likely minimizing dietary and environmental differences. Second, duodenal samples were taken when the subjects were in a fasting state, and intestinal function might vary over time (i.e., after meal assumption) [44]. Finally, the described obese-associated transcriptomic profile requires further metabolomics and/or proteomics validation. To the best of our knowledge, this is the first report on duodenal metatranscriptomic profiles in obese subjects. If our findings are confirmed in larger cohorts than ours, from various geographical areas and different lifestyles, we could hypothesize therapeutic strategies attempts (i.e., prebiotics, probiotics, or antibiotics) aimed at producing a desired change in microbial composition and/or function that favourably impacts the host metabolism, hopefully reducing obesity and the risk of obese-associated diseases. Furthermore, the evaluation of the influence of single nutrients on the obese-associated microbiota and microbiota-host interaction may provide further insights on diet-based treatments for the management of obese subjects.

\section{Conclusions}

Globally, we provided a global picture of duodenal transcriptome perturbations associated with severe obesity. Duodenum is a gastrointestinal region whose bacterial assemblage differs from that of other more frequently studied regions and scarcely investigated in obesity, notwithstanding its importance for the nutrients' digestion. We found that microbial composition and functional activity only partially coincided. Indeed, the carbohydrate and lipid metabolic pathways were among those most actively deregulated in the duodenum of obese subjects. Defects in human genes involved in epithelial intestinal cell differentiation, permeability function, and inflammation may also contribute to the obesity-associated gut dysbiosis.

Supplementary Materials: The following are available online at http://www.mdpi.com/2076-2607/8/11/1811/s1, Figure S1. Barplots showing the relative abundances of Prevotella, Streptococcus, Bacteroides genera and corresponding Bacteroidetes and Firmicutes phyla. The barplot on the right shows the values of OB and CO samples of the ratios Firmicutes/Bacteroidetes, Prevotella/Bacteroides and Streptococcus/Prevotella. The mean \pm SE is indicated for each bar. The significance of distribution differences has been calculated applying the Kruskal-Wallis test $\left({ }^{*} p<0.05 ;{ }^{* *} p<0.01\right)$; Supplementary File 1. Additional methodological details regarding samples collection, RNA isolation with technical controls and sequencing procedures; Supplementary File 2. Excel File containing the significant RefSeq functions (sheet 1), organisms (sheet 2) and functions specifically associated to Prevotella and Streptococcus genera (sheet 3). For each term, the corresponding $\log 2 \mathrm{FC}(\mathrm{OBvsCO})$ and adjusted $p$-value are also reported; Supplementary File 3. Excel File containing differentially expressed L3 (sheet1) and L4 (sheet2) SEED subsystem terms and their correspondent upper levels. Log2FC values and BH adjusted $p$-values are also reported. The different colors refer to L1 categorization; Supplementary File 4. Excel File containing up- (sheet1) 
and down- (sheet2) regulated host transcripts between OB and CO. For each Ensembl transcript id are reported the following annotations: the Ensembl gene Id, the Gene symbol, the Log2FC value, the BH adjusted $p$-value, the Gene name, the GO Biological Processes, the GO Molecular Functions and the KEGG pathways.; Table S1. Clinical and biochemical characteristics of lean (CO) and obese (OB) subjects.

Author Contributions: I.G. bioinformatics and statistical analysis of data, manuscript writing; C.N. execution of experiments, results analysis and interpretation, manuscript writing; V.D. contribution to manuscript writing; M.R.G. statistical consulting; V.P., S.T., D.C. and G.N. patient recruitment, sample and clinical data collection; L.S. study conception and design, manuscript writing, final revision and editing of the manuscript. All authors have read and agreed to the final version of the manuscript.

Funding: This research was funded by Research project SATIN (Sviluppo di Approcci Terapeutici Innovativi per patologie neoplastiche resistenti ai trattamenti) -D.D. n. 355 del 5/06/2017-Fondo FESR 2014/2020.

Acknowledgments: We thank Jean Ann Gilder (Scientific Communications srl., Naples, Italy) for language assistance, and Laura Iaffaldano and Giuseppe Labruna (CEINGE Biotecnologie Avanzate s. c. a r. 1., Naples, Italy) for technical support. We also thank Vittorio Lucignano, CEINGE-Biotecnologie Avanzate, for technical assistance related to graphics.

Conflicts of Interest: The authors declare no conflict of interest. The funders had no role in the design of the study; in the collection, analyses, or interpretation of data; in the writing of the manuscript, or in the decision to publish the results.

\section{Abbreviations}

GAPDH NAD-dependent glyceraldehyde-3-phosphate dehydrogenase (EC 1.2.1.12)

spxB Pyruvate oxidase (EC 1.2.3.3)

GDH NADP-specific glutamate dehydrogenase (EC 1.4.1.4)

PFL Pyruvate formate-lyase (EC 2.3.1.54)

pfkA 6-phosphofructokinase (EC 2.7.1.11)

PGK Phosphoglycerate kinase (EC 2.7.2.3)

lysC Aspartokinase (EC 2.7.2.4)

ppc Phosphoenolpyruvate carboxylase (EC 4.1.1.31)

FBA Fructose-bisphosphate aldolase class II (EC 4.1.2.13)

ENO Enolase (EC 4.2.1.11)

gln A Glutamine synthetase type I (EC 6.3.1.2)

murD UDP-N-acetylmuramoylalanine-D-glutamate ligase (EC 6.3.2.9)

ATPeF1G ATP synthase gamma chain (EC 3.6.3.14)

glgX Glycogen debranching enzyme (EC 3.2.1.196)

por Pyruvate-flavodoxin oxidoreductase (EC 1.2.7.1)

nrdD Ribonucleotide reductase of class III (anaerobic), large subunit (EC 1.17.4.2)

PIK3C2B phosphatidylinositol-4-phosphate 3-kinase catalytic subunit type 2 beta (EC 2.7.1.154)

PPT palmitoyl-protein thioesterase 1 (EC 3.1.2.22)

PDE4 phosphodiesterase 4 (EC 3.1.4.53)

ADH1B alcohol dehydrogenase 1B (class I), beta polypeptide (ADH1B) (EC 1.1.1.1)

ACP5 acid phosphatase 5, tartrate resistant (EC 3.1.3.2)

\section{References}

1. Finkelstein, E.A.; Khavjou, O.A.; Thompson, H.; Trogdon, J.G.; Pan, L.; Sherry, B.; Dietz, W. Obesity and Severe Obesity Forecasts Through 2030. Am. J. Prev. Med. 2012, 42, 563-570. [CrossRef] [PubMed]

2. Meijnikman, A.S.; Gerdes, V.E.; Nieuwdorp, M.; Herrema, H. Evaluating Causality of Gut Microbiota in Obesity and Diabetes in Humans. Endocr. Rev. 2018, 39, 133-153. [CrossRef] [PubMed]

3. Sacchetti, L.; Nardelli, C. Gut microbiome investigation in celiac disease: From methods to its pathogenetic role. Clin. Chem. Lab. Med. 2020, 58, 340-349. [CrossRef] [PubMed]

4. Hopkins, B.D.; Goncalves, M.D.; Cantley, L.C. Obesity and Cancer Mechanisms: Cancer Metabolism. J. Clin. Oncol. 2016, 34, 4277-4283. [CrossRef] [PubMed]

5. Biener, A.I.; Cawley, J.; Meyerhoefer, C. The Impact of Obesity on Medical Care Costs and Labor Market Outcomes in the US. Clin. Chem. 2018, 64, 108-117. [CrossRef] [PubMed] 
6. Finkelstein, E.A.; Trogdon, J.G.; Brown, D.S.; Allaire, B.T.; Dellea, P.S.; Kamal-Bahl, S.J. The Lifetime Medical Cost Burden of Overweight and Obesity: Implications for Obesity Prevention. Obesity 2008, 16, 1843-1848. [CrossRef]

7. Blakemore, A.I.; Froguel, P. Investigation of Mendelian forms of obesity holds out the prospect of personalized medicine. Ann. N. Y. Acad. Sci. 2010, 1214, 180-189. [CrossRef]

8. Rohde, K.; Keller, M.; Poulsen, L.L.C.; Blüher, M.; Kovacs, P.; Böttcher, Y. Genetics and epigenetics in obesity. Metabolism 2019, 92, 37-50. [CrossRef]

9. Mathes, W.F.; Kelly, S.A.; Pomp, D. Advances in comparative genetics: Influence of genetics on obesity. Br. J. Nutr. 2011, 106, S1-S10. [CrossRef]

10. Capobianco, V.; Nardelli, C.; Ferrigno, M.; Iaffaldano, L.; Pilone, V.; Forestieri, P.; Sacchetti, L. MiRNA and protein expression profiles of visceral adipose tissue reveal miR-141/ywhag and miR-520e/rab11a as two potential miRNA/protein target pairs associated with severe obesity. J. Proteome Res. 2012, 11, 3358-3369. [CrossRef]

11. Nardelli, C.; Granata, I.; Iaffaldano, L.; D’Argenio, V.; Del Monaco, V.; Maruotti, G.M.; Guarracino, M.R. MiR-138/miR-222 overexpression characterizes the miRNome of amniotic mesenchymal stem cells in obesity. Stem Cells Dev. 2017, 26, 4-14. [CrossRef] [PubMed]

12. Turnbaugh, P.J.; Hamady, M.; Yatsunenko, T.; Cantarel, B.L.; Duncan, A.; Ley, R.E.; Sogin, M.L.; Jones, W.J.; Roe, B.A.; Affourtit, J.P.; et al. A core gut microbiome in obese and lean twins. Nature 2009, 457, 480-484. [CrossRef] [PubMed]

13. Qin, J.; Li, R.; Raes, J.; Arumugam, M.; Burgdorf, K.S.; Manichanh, C.; Nielsen, T.; Pons, N.; Levenez, F.; Yamada, T.; et al. A human gut microbial gene catalogue established by metagenomic sequencing. Nat. Cell Biol. 2010, 464, 59-65. [CrossRef] [PubMed]

14. John, G.K.; Mullin, G.E. The Gut Microbiome and Obesity. Curr. Oncol. Rep. 2016, 18, 1-7. [CrossRef]

15. Sender, R.; Fuchs, S.; Milo, R. Are We Really Vastly Outnumbered? Revisiting the Ratio of Bacterial to Host Cells in Humans. Cell 2016, 164, 337-340. [CrossRef]

16. Alonso, V.R.; Guarner, F. Linking the gut microbiota to human health. Br. J. Nutr. 2013, 109, S21-S26. [CrossRef]

17. Kho, Z.Y.; Lal, S.K. The Human Gut Microbiome-A Potential Controller of Wellness and Disease. Front. Microbiol. 2018, 9, 1835. [CrossRef]

18. Goodwin, S.; McPherson, J.D.; McCombie, W.R. Coming of age: Ten years of next-generation sequencing technologies. Nat. Rev. Genet. 2016, 17, 333-351. [CrossRef]

19. Kuczynski, J.; Lauber, C.L.; Walters, W.A.; Parfrey, L.W.; Clemente, J.C.; Gevers, D.; Knight, R. Experimental and analytical tools for studying the human microbiome. Nat. Rev. Genet. 2012, 13, 47-58. [CrossRef]

20. Shakya, M.; Lo, C.-C.; Chain, P.S. Advances and Challenges in Metatranscriptomic Analysis. Front. Genet. 2019, 10, 904. [CrossRef]

21. Kootte, R.S.; Vrieze, A.; Holleman, F.; Dallinga-Thie, G.M.; Zoetendal, E.G.; De Vos, W.M.; Groen, A.K.; Hoekstra, J.B.L.; Stroes, E.S.; Nieuwdorp, M. The therapeutic potential of manipulating gut microbiota in obesity and type 2 diabetes mellitus. Diabetes Obes. Metab. 2011, 14, 112-120. [CrossRef] [PubMed]

22. Moran-Ramos, S.; López-Contreras, B.E.; Canizales-Quinteros, S. Gut Microbiota in Obesity and Metabolic Abnormalities: A Matter of Composition or Functionality? Arch. Med Res. 2017, 48, 735-753. [CrossRef] [PubMed]

23. Sun, L.; Ma, L.; Ma, Y.; Zhang, F.; Zhao, C.; Nie, Y. Insights into the role of gut microbiota in obesity: Pathogenesis, mechanisms, and therapeutic perspectives. Protein Cell 2018, 9, 397-403. [CrossRef] [PubMed]

24. Nardelli, C.; Granata, I.; D'Argenio, V.; Tramontano, S.; Compare, D.; Guarracino, M.R.; Nardone, G.; Pilone, V.; Sacchetti, L. Characterization of the Duodenal Mucosal Microbiome in Obese Adult Subjects by 16S rRNA Sequencing. Microorganisms 2020, 8, 485. [CrossRef]

25. Rothschild, D.; Weissbrod, O.; Barkan, E.; Kurilshikov, A.; Korem, T.; Zeevi, D.; Shilo, S. Environment dominates over host genetics in shaping human gut microbiota. Nature 2018, 555, 210-215. [CrossRef] [PubMed]

26. Schirmer, M.; Franzosa, E.A.; Lloyd-Price, J.; McIver, L.J.; Schwager, R.; Poon, T.W.; Ananthakrishnan, A.N.; Andrews, E.; Barron, G.; Lake, K.; et al. Dynamics of metatranscription in the inflammatory bowel disease gut microbiome. Nat. Microbiol. 2018, 3, 337-346. [CrossRef] 
27. Abu-Ali, G.S.; Mehta, R.S.; Lloyd-Price, J.; Mallick, H.; Branck, T.; Ivey, K.L.; Drew, D.A.; Dulong, C.; Rimm, E.; Izard, J.; et al. Metatranscriptome of human faecal microbial communities in a cohort of adult men. Nat. Microbiol. 2018, 3, 356-366. [CrossRef]

28. Wang, M.; Ahrné, S.; Jeppsson, B.; Molin, G. Comparison of bacterial diversity along the human intestinal tract by direct cloning and sequencing of $16 \mathrm{~S}$ rRNA genes. FEMS Microbiol. Ecol. 2005, 54, 219-231. [CrossRef]

29. Vasapolli, R.; Schütte, K.; Schulz, C.; Vital, M.; Schomburg, D.; Pieper, D.H.; Vilchez-Vargas, R.; Malfertheiner, P. Analysis of Transcriptionally Active Bacteria Throughout the Gastrointestinal Tract of Healthy Individuals. Gastroenterology 2019, 157, 1081-1092. [CrossRef]

30. Zoetendal, E.G.; Raes, J.; Bogert, B.V.D.; Arumugam, M.; Booijink, C.C.G.M.; Troost, F.J.; Bork, P.; Wels, M.; De Vos, W.M.; Kleerebezem, M. The human small intestinal microbiota is driven by rapid uptake and conversion of simple carbohydrates. ISME J. 2012, 6, 1415-1426. [CrossRef]

31. Krajmalnik-Brown, R.; Ilhan, Z.-E.; Kang, D.-W.; DiBaise, J.K. Effects of Gut Microbes on Nutrient Absorption and Energy Regulation. Nutr. Clin. Pract. 2012, 27, 201-214. [CrossRef] [PubMed]

32. Tomé, D. Digestibility Issues of Vegetable versus Animal Proteins: Protein and Amino Acid Requirements-Functional Aspects. Food Nutr. Bull. 2013, 34, 272-274. [CrossRef] [PubMed]

33. Andrews, S. FASTQC. A Quality Control Tool for High Throughput Sequence Data. 2010. Available online: https://www.scienceopen.com/document?vid=de674375-ab83-4595-afa9-4c8aa9e4e736 (accessed on 16 November 2020).

34. Bolger, A.M.; Lohse, M.; Usadel, B. Trimmomatic: A flexible trimmer for Illumina sequence data. Bioinformatics 2014, 30, 2114-2120. [CrossRef]

35. Langmead, B.; Salzberg, S.L. Fast gapped-read alignment with Bowtie 2. Nat. Methods 2012, 9, 357-359. [CrossRef] [PubMed]

36. Bray, N.L.; Pimentel, H.; Melsted, P.; Pachter, L. Near-optimal probabilistic RNA-seq quantification. Nat. Biotechnol. 2016, 34, 525-527. [CrossRef] [PubMed]

37. Love, M.H.; Huber, W.; Anders, S. Moderated estimation of fold change and dispersion for RNA-seq data with DESeq2. Genome Biol. 2014, 15, 550. [CrossRef] [PubMed]

38. Kopylova, E.; Noé, L.; Touzet, H. SortMeRNA: Fast and accurate filtering of ribosomal RNAs in metatranscriptomic data. Bioinformatics 2012, 28, 3211-3217. [CrossRef] [PubMed]

39. Buchfink, B.; Xie, C.; Huson, D.H. Fast and sensitive protein alignment using DIAMOND. Nat. Methods 2015, 12, 59-60. [CrossRef] [PubMed]

40. Westreich, S.T.; Treiber, M.L.; Mills, D.A.; Korf, I.; Lemay, D.G. SAMSA2: A standalone metatranscriptome analysis pipeline. BMC Bioinform. 2018, 19, 175. [CrossRef]

41. Franzosa, E.A.; McIver, L.J.; Rahnavard, G.; Thompson, L.R.; Schirmer, M.; Weingart, G.; Lipson, K.S.; Knight, R.; Caporaso, J.G.; Segata, N.; et al. Species-level functional profiling of metagenomes and metatranscriptomes. Nat. Methods 2018, 15, 962-968. [CrossRef]

42. Caspi, R.; Billington, R.; Keseler, I.M.; Kothari, A.; Krummenacker, M.; E Midford, P.; Ong, W.K.; Paley, S.; Subhraveti, P.; Karp, P.D. The MetaCyc database of metabolic pathways and enzymes-A 2019 update. Nucleic Acids Res. 2020, 48, D445-D453. [CrossRef] [PubMed]

43. Mallick, H.; Rahnavard, A.; McIver, L. Maaslin2: Maaslin2. R Package Version 1.2.0. Bioconductor. 2019. Available online: http://huttenhower.sph.harvard.edu/maaslin2 (accessed on 10 November 2020). [CrossRef]

44. Sanchez, A.; Golding, I. Genetic Determinants and Cellular Constraints in Noisy Gene Expression. Science 2013, 342, 1188-1193. [CrossRef] [PubMed]

45. D'Souza, G.; Kost, C. Experimental Evolution of Metabolic Dependency in Bacteria. PLoS Genet. 2016, 12, e1006364. [CrossRef] [PubMed]

46. Ley, R.E.; Bäckhed, F.; Turnbaugh, P.; Lozupone, C.A.; Knight, R.D.; Gordon, J.I. Obesity alters gut microbial ecology. Proc. Natl. Acad. Sci. USA 2005, 102, 11070-11075. [CrossRef]

47. Ley, R.E.; Turnbaugh, P.J.; Klein, S.; Gordon, J.I. Human gut microbes associated with obesity. Nat. Cell Biol. 2006, 444, 1022-1023. [CrossRef]

48. Ley, R.E. Prevotella in the gut: Choose carefully. Nat. Rev. Gastroenterol. Hepatol. 2016, 13, 69-70. [CrossRef]

49. Gorvitovskaia, A.; Holmes, S.P.; Huse, S.M. Interpreting Prevotella and Bacteroides as biomarkers of diet and lifestyle. Microbiome 2016, 4, 15. [CrossRef]

50. Arumugam, M.; Raes, J.; Pelletier, E.; Le Paslier, D.; Yamada, T.; Mende, D.R.; Fernandes, G.R.; Tap, J.; Bruls, T.; Batto, J.M.; et al. Enterotypes of the human gut microbiome. Nature 2011, 473, 174-180. [CrossRef] 
51. De Filippis, F.; Pellegrini, N.; Vannini, L.; Jeffery, I.B.; La Storia, A.; Laghi, L.; I Serrazanetti, D.; Di Cagno, R.; Ferrocino, I.; Lazzi, C.; et al. High-level adherence to a Mediterranean diet beneficially impacts the gut microbiota and associated metabolome. Gut 2015, 65, 1812-1821. [CrossRef]

52. Hjorth, M.F.; Christensen, L.; Kjølbæk, L.; Larsen, L.H.; Roager, H.M.; Kiilerich, P.; Kristiansen, K.; Astrup, A. Pretreatment Prevotella-to-Bacteroides ratio and markers of glucose metabolism as prognostic markers for dietary weight loss maintenance. Eur. J. Clin. Nutr. 2019, 74, 338-347. [CrossRef] [PubMed]

53. Franke, T.; Deppenmeier, U. Physiology and central carbon metabolism of the gut bacterium Prevotella copri. Mol. Microbiol. 2018, 109, 528-540. [CrossRef] [PubMed]

54. De Angelis, M.; Ferrocino, I.; Calabrese, F.M.; De Filippis, F.; Cavallo, N.; Siragusa, S.; Rampelli, S.; Di Cagno, R.; Rantsiou, K.; Vannini, L.; et al. Diet influences the functions of the human intestinal microbiome. Sci. Rep. 2020, 10, 4247. [CrossRef] [PubMed]

55. De Filippis, F.; Pellegrini, N.; Laghi, L.; Gobbetti, M.; Ercolini, D. Unusual sub-genus associations of faecal Prevotella and Bacteroides with specific dietary patterns. Microbiome 2016, 4, 57. [CrossRef] [PubMed]

56. Dadashipour, M.; Iwamoto, M.; Hossain, M.M.; Akutsu, J.-I.; Zhang, Z.; Kawarabayasi, Y.; Hossain, M. Identification of a Direct Biosynthetic Pathway for UDP-N-Acetylgalactosamine from Glucosamine-6-Phosphate in Thermophilic Crenarchaeon Sulfolobus tokodaii. J. Bacteriol. 2018, 200, e00048-18. [CrossRef] [PubMed]

57. Sweeney, R.P.; Lowary, T.L. New insights into lipopolysaccharide assembly and export. Curr. Opin. Chem. Biol. 2019, 53, 37-43. [CrossRef] [PubMed]

58. Cani, P.D.; Possemiers, S.; Van De Wiele, T.; Guiot, Y.; Everard, A.; Rottier, O.; Geurts, L.; Naslain, D.; Neyrinck, A.; Lambert, D.M.; et al. Changes in gut microbiota control inflammation in obese mice through a mechanism involving GLP-2-driven improvement of gut permeability. Gut 2009, 58, 1091-1103. [CrossRef] [PubMed]

59. Clark, S.E.; Snow, J.; Li, J.; Zola, T.A.; Weiser, J.N. Phosphorylcholine Allows for Evasion of Bactericidal Antibody by Haemophilus influenzae. PLoS Pathog. 2012, 8, e1002521. [CrossRef] [PubMed]

60. Muscogiuri, G.; Cantone, E.; Cassarano, S.; Tuccinardi, D.; Barrea, L.; Savastano, S.; Colao, A. Gut microbiota: A new path to treat obesity. Int. J. Obes. Suppl. 2019, 9, 10-19. [CrossRef]

61. Zhao, F.-J. A novel pathway of arsenate detoxification. Mol. Microbiol. 2016, 100, 928-930. [CrossRef]

62. Chen, J.; Yoshinaga, M.; Garbinski, L.D.; Rosen, B.P. Synergistic interaction of glyceraldehydes-3-phosphate dehydrogenase and ArsJ, a novel organoarsenical efflux permease, confers arsenate resistance. Mol. Microbiol. 2016, 100, 945-953. [CrossRef] [PubMed]

63. Bekebrede, A.F.; Keijer, J.; Gerrits, W.J.J.; De Boer, V.C.J. The Molecular and Physiological Effects of Protein-Derived Polyamines in the Intestine. Nutrients 2020, 12, 197. [CrossRef] [PubMed]

64. Lipowska, J.; Miks, C.D.; Kwon, K.; Shuvalova, L.; Zheng, H.; Lewiński, K.; Cooper, D.R.; Shabalin, I.G.; Minor, W. Pyrimidine biosynthesis in pathogens-Structures and analysis of dihydroorotases from Yersinia pestis and Vibrio cholerae. Int. J. Biol. Macromol. 2019, 136, 1176-1187. [CrossRef] [PubMed]

65. Almohazey, D.; Lo, Y.-H.; Vossler, C.V.; Simmons, A.J.; Hsieh, J.J.; Bucar, E.B.; Schumacher, M.A.; E Hamilton, K.; Lau, K.S.; Shroyer, N.F.; et al. The ErbB3 receptor tyrosine kinase negatively regulates Paneth cells by PI3K-dependent suppression of Atoh1. Cell Death Differ. 2017, 24, 855-865. [CrossRef]

66. Sehgal, A.; Donaldson, D.S.; Pridans, C.; Sauter, K.A.; Hume, D.A.; Mabbott, N.A. The role of CSF1R-dependent macrophages in control of the intestinal stem-cell niche. Nat. Commun. 2018, 9, 1-17. [CrossRef]

67. Sato, T.; Van Es, J.H.; Snippert, H.J.; Stange, D.E.; Vries, R.G.; Born, M.V.D.; Barker, N.; Shroyer, N.F.; Van De Wetering, M.; Clevers, H. Paneth cells constitute the niche for Lgr5 stem cells in intestinal crypts. Nat. Cell Biol. 2011, 469, 415-418. [CrossRef]

68. Grewal, T.; Enrich, C.; Rentero, C.; Buechler, C. Annexins in Adipose Tissue: Novel Players in Obesity. Int. J. Mol. Sci. 2019, 20, 3449. [CrossRef]

69. Akasheh, R.T.; Pini, M.; Pang, J.; Fantuzzi, G. Increased Adiposity in Annexin A1-Deficient Mice. PLoS ONE 2013, 8, e82608. [CrossRef]

70. Kosicka, A.; Cunliffe, A.D.; MacKenzie, R.; Zariwala, M.G.; Perretti, M.; Flower, R.J.; Renshaw, D. Attenuation of plasma annexin A1 in human obesity. FASEB J. 2012, 27, 368-378. [CrossRef] 
71. Pietrani, N.T.; Ferreira, C.N.; Rodrigues, K.F.; Perucci, L.O.; Carneiro, F.S.; Bosco, A.A.; Oliveira, M.C.; Pereira, S.S.; Teixeira, A.L.; Alvarez-Leite, J.I.; et al. Proresolving protein Annexin A1: The role in type 2 diabetes mellitus and obesity. Biomed. Pharmacother. 2018, 103, 482-489. [CrossRef]

72. Purvis, G.S.D.; Collino, M.; Loiola, R.A.; Baragetti, A.; Chiazza, F.; Brovelli, M.; Sheikh, M.H.; Collotta, D.; Cento, A.; Mastrocola, R.; et al. Identification of AnnexinA1 as an Endogenous Regulator of RhoA, and Its Role in the Pathophysiology and Experimental Therapy of Type-2 Diabetes. Front. Immunol. 2019, 10, 571. [CrossRef] [PubMed]

73. Nelson, W.J. Epithelial cell polarity from the outside looking in. Physiology 2003, 18, 143-146. [CrossRef] [PubMed]

74. Tanos, B.E.; Yeaman, C.; Rodriguez-Boulan, E. An emerging role for IQGAP1 in tight junction control. Small GTPases 2018, 9, 375-383. [CrossRef] [PubMed]

75. Li, Z.; Kim, S.H.; Higgins, J.M.G.; Brenner, M.B.; Sacks, D.B. IQGAP1 and Calmodulin Modulate E-cadherin Function. J. Biol. Chem. 1999, 274, 37885-37892. [CrossRef] [PubMed]

76. Lechuga, S.; Baranwal, S.; Ivanov, A.I. Actin-interacting protein 1 controls assembly and permeability of intestinal epithelial apical junctions. Am. J. Physiol. Liver Physiol. 2015, 308, G745-G756. [CrossRef] [PubMed]

77. Moir, R.; Spann, T. The structure and function of nuclear lamins: Implications for disease. Cell. Mol. Life Sci. 2001, 58, 1748-1757. [CrossRef]

78. Wong, C.-M.; Xu, L.; Yau, M.Y.-C. Alternative mRNA Splicing in the Pathogenesis of Obesity. Int. J. Mol. Sci. 2018, 19, 632. [CrossRef]

79. Winnier, D.A.; Fourcaudot, M.; Norton, L.; Abdul-Ghani, M.A.; Hu, S.L.; Farook, V.S.; Coletta, D.K.; Kumar, S.; Puppala, S.; Chittoor, G.; et al. Transcriptomic Identification of ADH1B as a Novel Candidate Gene for Obesity and Insulin Resistance in Human Adipose Tissue in Mexican Americans from the Veterans Administration Genetic Epidemiology Study (VAGES). PLoS ONE 2015, 10, e0119941. [CrossRef]

80. Ahtiainen, L.; Kolikova, J.; Mutka, A.-L.; Luiro, K.; Gentile, M.; Ikonen, E.; Khiroug, L.; Jalanko, A.; Kopra, O. Palmitoyl protein thioesterase 1 (Ppt1)-deficient mouse neurons show alterations in cholesterol metabolism and calcium homeostasis prior to synaptic dysfunction. Neurobiol. Dis. 2007, 28, 52-64. [CrossRef]

81. Zhao, W.; Su, J.; Wang, Y.; Qian, T.; Liu, Y. Functional importance of palmitoyl protein thioesterase 1 (PPT1) expression by Sertoli cells in mediating cholesterol metabolism and maintenance of sperm quality. Mol. Reprod. Dev. 2019, 86, 984-998. [CrossRef]

82. Margaria, J.P.; Ratto, E.; Gozzelino, L.; Li, H.; Hirsch, E. Class II PI3Ks at the Intersection between Signal Transduction and Membrane Trafficking. Biomolecules 2019, 9, 104. [CrossRef] [PubMed]

Publisher's Note: MDPI stays neutral with regard to jurisdictional claims in published maps and institutional affiliations.

(C) 2020 by the authors. Licensee MDPI, Basel, Switzerland. This article is an open access article distributed under the terms and conditions of the Creative Commons Attribution (CC BY) license (http://creativecommons.org/licenses/by/4.0/). 